\title{
IMPULSIVE CONTROL OF AN INTEGRATED PEST MANAGEMENT MODEL WITH DISPERSAL BETWEEN PATCHES
}

\author{
PAUL GEORGESCU* \\ Department of Mathematics \\ "Gheorghe Asachi" Technical University of Iaşi \\ Bd. Copou 11, 700506 Iaşi, Romania \\ v.p.georgescu@gmail.com \\ GABRIEL DIMITRIU \\ Department of Mathematics and Informatics \\ "Grigore T. Popa" University of Medicine and Pharmacy \\ Bd. Universităţii 16, Iaşi, 700115 Iaşi, Romania \\ dimitriu.gabriel@gmail.com \\ ROBERT SINCLAIR \\ Mathematical Biology Unit \\ Okinawa Institute of Science and Technology \\ 1919-1 Tancha, Onna-Son, Okinawa 904-0412, Japan \\ sinclair@oist.jp \\ Received 20 December 2009 \\ Accepted 23 June 2010
}

\begin{abstract}
We consider a two-patch $S I$ model of integrated pest management with dispersal of both susceptible and infective pests between patches. A biological control, consisting of the periodic release of infective pests and a chemical control, consisting of periodic and impulsive pesticide spraying, are employed in order to maintain the size of the pest population below an economically acceptable level. It is assumed that the spread of the disease which is inflicted on the pest population through the use of the biological control is characterized by a nonlinear force of infection expressed in an abstract form. A sufficient condition for the local stability of the susceptible pest-eradication periodic solution is found using Floquet theory for periodic systems of ordinary differential equations, an analysis of the influence of dispersal between patches being performed for several particular cases. Our numerical simulations indicate that an increase in the amount but not in the frequency of pesticide use may not result in control. We also show that patches which are stable in isolation can be destabilized by dispersal between patches.
\end{abstract}

Keywords: Integrated Pest Management; SI Model; Impulsive Controls; Patch Structure; Population Dispersal; Nonlinear Infection Rate; Stability; Floquet Theory.

${ }^{*}$ Corresponding author. 


\section{Introduction}

The environmental and health risks caused by the indiscriminate use or misuse of pesticides are now well-documented. It has been shown that overexposure to pesticides is associated with chronic health problems ${ }^{1,2}$ and often human poisoning, ${ }^{3}$ as well as a general decrease in the biodiversity of the environment. ${ }^{4}$ The urgency of the need for alternatives is further illustrated by the fact that the development of resistance to even the strongest pesticides, such as DDT, ${ }^{5}$ can occur. ${ }^{6}$ Consequently, Integrated Pest Management (IPM) has emerged as an integrative, sustainable and environmentally friendly approach which employs a large array of combined techniques in order to control pests while minimizing hazardous effects on non-target organisms. Here, "pests" refers to any organisms which are detrimental to human interests, including vertebrate or invertebrate animals, pathogens and weeds, while "management" refers to a set of decision rules based on ecological as well as on economical principles. ${ }^{7}$ One does not usually aim to eradicate pests completely, as this would not be cost-effective and would unnecessarily damage the ecosystem, but to reduce the pest population under an economically significant level called the economic injury level (EIL). ${ }^{8}$ In this approach, pesticides are used only when they are deemed as an absolute necessity, as they are sometimes the quickest way to contain a pest outbreak, the emphasis being put on the use of biological and mechanical controls, as well as preventive cultural practices.

Biological control of insects involves the use of natural enemies (predators, parasitoids or pathogens) or natural pesticides such as $\mathrm{Bt}^{9,10}$ to reduce or maintain pest populations under EIL. ${ }^{11}$ Several examples of successful application of biological control are the use of the predatory arthropod Orius sauteri to protect eggplant crops in greenhouses in Japan against the pest Thrips palmi Karny (melon thrips), ${ }^{12}$ the use of the predatory mites Phytoseiulus persimilis and Neoseiulus californicus against the red spider mite Tranychus urticae Koch in strawberry fields in England ${ }^{13}$ and the use of granuloviruses against the diamondback moth Plutella xylostella in cabbage farms in East Africa. ${ }^{14}$ See Ref. 15 for an overview of how biocontrol agents act towards the incapacitation of pests.

Among the possible approaches to biological control we mention conservation, importation and augmentation methods. The goal of the conservation method is to reduce the factors which interfere with the growth of the natural enemies of the given pests and to provide resources that natural enemies rely upon, ${ }^{16}$ while the purpose of the importation method is to reunite prey, that have become pests outside their original geographical areas, with their natural predators. ${ }^{17}$

Augmentation represents the manipulation of natural enemy populations in order to increase their effectiveness. ${ }^{11} \mathrm{~A}$ typical approach to augmentation consists of raising and periodically releasing natural enemies into the environments where pest suppression is needed. This may take the form of inoculative release, in which the natural enemy is intended to establish and reproduce in the environment, while keeping the pest in control, or the form of inundative release, in 
which large amounts of natural enemies are released for the purpose of overwhelming the pest, the persistence of the natural enemies in the environment not being of concern.

A variant of the innoculative method is to periodically release infective pest individuals, with the purpose of maintaining the endemicity of a disease into the target pest population, on the grounds that infective pests are less likely to reproduce and to damage the environment. In this case, the dynamics of the pest population under consideration may be described using a compartmental model for disease propagation. The inherent discontinuity of the control activities and the immediate jumps in the size of the infective pest population after each release can be described using models involving impulsive perturbations. See Refs. 18-20 for a general overview of impulsive control theory and of its applications, as well as Refs. 21-23 for other impulsive models of IPM. An analysis of optimal timing for impulsive IPM models has been performed by Tang, Tang and Cheke. ${ }^{24}$ See also Refs. 25 and 26 for optimal control problems associated with a three-dimensional food chain and to a Lotka-Volterra model, respectively.

Ultimately, all species live in environments which are patched at one scale or another. Patchiness may occur since niches, conditions and food resources which favor survival are unevenly distributed in space and time, facilitating aggregation of individuals. ${ }^{27}$ Patchiness may also be a feature of the physical environment itself, which may consist of separate spaces (islands, mountain tops) surrounded by inhospitable areas. Finally, the patch structure may be an outcome of the activities of the organisms themselves, through the depletion of local resources.

Consequently, an approach to spatial modelling is to consider spatially heterogeneous groups of populations (metapopulations) which are connected by dispersal. Among factors which favor population migration between patches are mating and breeding, seasonal or diurnal variability of the environment, avoidance of overcrowding, inbreeding and kin competition. ${ }^{28}$ Dispersal rate is sometimes considered a characteristic trait of a species, as dispersal may occur at a constant per-capita rate, but might also be condition-dependent, in particular density-dependent. Dispersal may either increase (positive density-dependent dispersal) or decrease (negative density-dependent dispersal) as population density increases, both forms of dispersal being observed in mites, insects and vertebrates. ${ }^{29}$

Mathematical models of interacting populations which disperse between patches have become a subject of growing interest (see, for instance Refs. 30-35). Most papers focus on discussing the coexistence or extinction of species, or the existence and stability of the positive steady states. However, the stability results obtained therein are usually of a local nature, due to the complexity of the global analysis. The influence of dispersal upon the stability of the equilibria remains largely unknown, again due to computational difficulties. In this regard, it has been observed that while dispersal on its own is not stabilizing and can destabilize local equilibria, its connection with other features such as spatial and temporal 
heterogeneity can promote stability (see the comprehensive survey of Briggs and Hoopes) ${ }^{36}$ while it has also been observed that under some particular conditions, density dependent dispersal can generate limit cycles. ${ }^{37}$

The purpose of this paper is to construct a two-patch SI model of IPM with dispersal of both susceptible and infective pests between patches, which is subject to impulsive and periodic biological and chemical controls, employed with the same periodicity, but not simultaneously. It is assumed that the biological control consists of the periodic release of infective pests in a constant amount, while the chemical control consists of periodic pesticide spraying, which causes the removal of fixed proportions of susceptible and infective pests. The spread of the disease which is inflicted on the pest population through the release of infective pests is characterized by a nonlinear force of infection given in an abstract, unspecified form. Of concern is the stability of the susceptible pest-eradication periodic solution, a sufficient condition for local stability being obtained via the use of Floquet theory for periodic systems of ordinary differential equations. Our results extend those in Ref. 38, where a single-patch version of our model is discussed, and those in Ref. 39, where only susceptible pests were allowed to disperse between patches.

The remaining part of this paper is organized as follows: In Sec. 2, our twopatch model is formulated together with the biological assumptions it relies upon. Several stability, persistence and bifurcation results which are valid for the singlepatch version of this model are also listed for future reference. In Sec. 3, a few basic notions regarding Floquet theory for periodic systems of linear differential equations are introduced together with a discussion of time-dependent matrix exponentials. Section 4 is concerned with proving the existence and global attractivity of a periodic solution for the reduced (no susceptible pests) system. In Sec. 5, the stability properties of the corresponding susceptible pest-eradication state are discussed under general assumptions. Our results are then specialized for several particular cases, the influence of dispersal between patches upon the stability of the susceptible pest-eradication state being then investigated. Several comments regarding the biological significance of our results are also formulated. In particular, we are able to show analytically that the dispersal of pests has the potential to destabilize an otherwise stable system.

In Sec. 6, we provide the results of numerical simulations which illustrate various properties of our model. By simulation, we are also able to go beyond our analytical results and show what appear to be period-doubling bifurcations leading to $\operatorname{chaos}^{40}$ in the one-patch model in its unstable regime. For this model, we are able to show that an increase in the amount, but not frequency, of application of pesticides will not always lead to control, a result with obvious biological significance. Finally, we present a numerical example of two stable patches being destabilized by pest dispersal, a result, consistent with earlier theoretical findings, ${ }^{41-43}$ of some importance for any attempt to understand the application of IPM to patched landscapes. 


\section{The Model}

In the following, we shall suppose that the environment consists of two distinct patches, $P_{1}$ and $P_{2}$ and denote by $S_{i}$ and $I_{i}$ the sizes of the susceptible pest population and infective pest population in patch $i$, respectively $(i=1,2)$. The following assumptions are made to derive our mathematical model:

(A1) All pests are either susceptible or infective. The infective pests may neither damage the crop, reproduce nor recover.

(A2) The incidence rate of infection in patch $i$ is given by $g\left(I_{i}\right) S_{i}, i=1,2$, where $g$ is the (possibly nonlinear) force of infection, assumed to be the same in each patch.

(A3) In the absence of infection, the intrinsic growth rate of the susceptible class $S_{i}$ is given by the logistic-like growth function $S_{i} b_{i}\left(S_{i}\right)$, where $b_{i}$ are (possibly nonlinear) birth functions, $i=1,2$.

(A4) Infective pests are impulsively released in both patches with periodicity $T>0$, in the same amount $\mu>0$ each time.

(A5) The mortality rate of the infective population $I_{i}$ is $w_{i}>0, i=1,2$. The time scale of the disease propagation is assumed to be fast enough that the natural mortality of susceptibles need not be considered.

(A6) Pesticides are sprayed with periodicity $T$ and with a fixed efficiency, in the sense that fixed proportions $\delta_{1}$ and $\delta_{2}$ of the susceptible pest populations $S_{1}$ and $S_{2}$, respectively, and a fixed proportion $\delta_{I}$ of the infective pest populations $I_{1}$ and $I_{2}, 0<\delta_{1}, \delta_{2}, \delta_{I}<1$, are removed each time the pesticides are applied.

(A7) All pests, susceptible or infective, can disperse between patches.

The birth functions $b_{1}, b_{2}$ and the force of infection $g$ are assumed to satisfy the following hypotheses:

(B) $b_{i}(0)=r_{i}, b_{i}$ is decreasing on $[0, \infty), \lim _{S \rightarrow \infty} b_{i}(S)<-w_{i}, S \mapsto S b_{i}(S)$ is locally Lipschitz on $(0, \infty), i=1,2$.

(G) $g(0)=0, g$ is increasing and globally Lipschitz on $[0, \infty)$.

Note that hypothesis $(B)$ is satisfied if the growth rates of the susceptible pest populations $S_{i}$ are given by the Richards growth law $\left(S_{i} b\left(S_{i}\right)=\frac{r_{R}}{1-p} S_{i}\left(\left(\frac{S_{i}}{K}\right)^{p}-1\right)\right.$, $p \neq 1$ ), which generalizes the logistic growth law. Regarding the propagation of the disease which is spread through the periodic release of infective pests, it has been observed (see Refs. 44 and 45) that the dependence of the rate of incidence of infection upon the size of the infective pest population plays a more significant role than the dependence upon the size of the susceptible pest population, which justifies the use of incidence rates of infection of type $g\left(I_{i}\right) S_{i}$. A nonlinear force of infection $g(I)=\frac{k I}{1+\alpha I}$ has been employed by Capasso and Serio. ${ }^{46}$ A very general incidence rate of type $g(S)=\frac{k I^{p}}{1+\alpha I^{q}}$ has been proposed by Liu, Levin and Iwasa; ${ }^{47}$ 
see also Ref. 48. Particular incidence rates of type $g(I)=\frac{k I^{2}}{1+\alpha I^{2}}, g(I)=\frac{k I}{1+\alpha I^{2}}$ have been used in Ruan and Wang, ${ }^{49}$ respectively in Xiao and Ruan. ${ }^{50}$

Assumption (A1) demands pure horizontal transmission of the infection, and this needs to be justified, particularly since purely vertical transmission of insect viruses is known, ${ }^{51}$ some parasites alternate between vertical and horizontal transmission as they move from one host to another ${ }^{52}$ and strategies requiring change from a vertical to a horizontal transmission mode in response to host stress are well documented in phage ${ }^{53}$ and also appear to have been adopted by honey bee viruses. ${ }^{54}$ Regarding diseases with very high vertical transmission, Wolbachia represents a maternally inherited proteobacteria which infects a wide range of arthropods, ${ }^{55}$ causing reproductive alterations in their hosts, such as cytoplasmatic incompatibility, parthenogenesis and feminization, all of which influence the population dynamics of Wolbachia as well as their hosts. Wolbachia is able to infect the reproductive tissues of arthropods and is transmitted to offspring via egg cytoplasm, which results in a very high transmission probability. Also, vertical transmission of viruses infectious to the tobacco budworm Heliothis virescens, the nucleopolyhedrosis viruses ${ }^{56}$ Autographa californica and Heliothis zea have been studied by Nordin, Brown and Jackson, ${ }^{57}$ being observed that all eggs laid by females indirectly contaminated with viruses had some degree of virus contamination, although much variation in concentration occured.

In other words, in order to understand natural pest control mechanisms fully, vertical transmission must be considered. We justify our restriction to horizontal transmission in our model formulation in two ways. First, pest control by disease can only be achieved by effective pathogenicity (virulence), and it is generally accepted that vertical transmission is typically associated with benign infection ${ }^{51}$ whereas horizontal transmission is associated with increased virulence. ${ }^{58-60}$ Our second reason for restricting our model to horizontal transmission only is the socioeconomic importance of damage caused by the diamondback moth Plutella xylostella, ${ }^{6,61}$ which affects cruciferous vegetable crops, and the potato tuberworm Phthorimaea operculella. ${ }^{62}$ It is the larvae of these pests which cause the damage. Our model is intended to be useful in understanding the control of these serious pests, taking the associated granuloviruses ${ }^{56,63} \mathrm{PxGV}^{14,64}$ and $\mathrm{PoGV}^{65}$ as promising biological control agents. These and other granuloviruses infect primarily larvae, and, among other things, induce secretion of an enzyme which inactivates a hormone needed to initiate pupation. ${ }^{66,67}$ The end effect is that the infected larvae are literally liquefied before they can pupate, and are thus prevented from becoming adults and reproducing.

Assumption (A6) should not mislead the reader into thinking that pesticides' effectiveness does not change with time, ${ }^{68}$ or that our model absolutely requires this. The assumption is perhaps best understood as referring to a steadily changing regime of pesticides, since this can approximate, for some limited time, the ideal of the pesticide for which there could be no resistance. 
Assumption (A7) does not necessarily imply that infective pests are able to move on their own accord between patches. Baculoviruses liquefy larvae, as already discussed above, and there is no reason to believe that infected larvae would be able to move to a new patch on their own. Infected larvae do however have a tendency to move up their host plant, and it has been suggested that this would in fact promote interpatch dispersal, since larvae become more visible to predators the higher up they are ${ }^{56}$ and predators can effectively disperse viruses from infected prey. ${ }^{69}$

As a result, the following impulsively perturbed system is formulated to describe the dynamics of the model under consideration:

$$
\left\{\begin{array}{rlrl}
S_{1}^{\prime}(t)= & S_{1}(t) b_{1}\left(S_{1}(t)\right)-g\left(I_{1}(t)\right) S_{1}(t) & & \\
& +d_{21} S_{2}(t)-d_{12} S_{1}(t), & & t \neq(n+l-1) T, t \neq n T \\
S_{2}^{\prime}(t)= & S_{2}(t) b_{2}\left(S_{2}(t)\right)-g\left(I_{2}(t)\right) S_{2}(t) & & \\
& +d_{12} S_{1}(t)-d_{21} S_{2}(t), & & t \neq(n+l-1) T, t \neq n T \\
I_{1}^{\prime}(t)= & g\left(I_{1}(t)\right) S_{1}(t)-w_{1} I_{1}(t) & & \\
& +D_{21} I_{2}(t)-D_{12} I_{1}(t), & & t \neq(n+l-1) T, t \neq n T \\
I_{2}^{\prime}(t)= & g\left(I_{2}(t)\right) S_{2}(t)-w_{2} I_{2}(t) & & \\
& +D_{12} I_{1}(t)-D_{21} I_{2}(t), & & t \neq(n+l-1) T, t \neq n T \\
\Delta S_{1}(t)= & -\delta_{1} S_{1}(t), & & t=(n+l-1) T \\
\Delta S_{2}(t)= & -\delta_{2} S_{2}(t), & & t=(n+l-1) T \\
\Delta I_{i}(t)= & -\delta_{I} I_{i}(t), & & t=(n+l-1) T, i=1,2 \\
\Delta S_{i}(t)= & 0, & & t=n T, i=1,2 \\
\Delta I_{i}(t)= & \mu, & t=n T, i=1,2 .
\end{array}\right.
$$

$\Delta \varphi(t)=\varphi(t+)-\varphi(t)$ for $\varphi \in\left\{S_{i}, I_{i}\right\}, i=1,2$ represent the instantaneous jumps in the sizes of the susceptible and infective pest populations, respectively, after the use of the impulsive controls. Note that these terms may also be used to describe the effects of selective catching or to conflate other regulatory measures rather than to describe pesticide spraying and pest release alone. Also, $l T, 0<l<1$, describes the time lag between the release of infective pests and pesticide spraying. The nonnegative constants $d_{i j}$ and $D_{i j}(i, j \in\{1,2\}, i \neq j)$ represent the dispersal rates of susceptibles and infectives from patch $i$ to patch $j$, respectively. Regarding pest dispersal between patches, a common situation is the one in which $d_{12}=d_{21}=d$ and $D_{12}=D_{21}=D$, i.e., the dispersal of susceptible pests and infective pests between patches obeys a Fickian law, being proportional to the difference between the respective population sizes.

This model has also been studied in Ref. 39 for $D_{12}=D_{21}=0$, i.e., in the situation in which only the susceptible pests are allowed to disperse, and a sufficient 
condition for the local stability of the susceptible pest-eradication periodic solution has been found via Floquet theory, the effect of pest dispersal between patches upon the stability of this solution also being discussed. The impulsive control of a twopatch Lotka-Volterra model has been considered by Yang and Tang, ${ }^{43}$ the effects of population dispersal upon pest persistence also being investigated. A single-patch version of this model has been considered by Georgescu and Moroşanu ${ }^{38}$ and by Georgescu, Zhang and Chen, ${ }^{70}$ in the following form:

$$
\left\{\begin{aligned}
S^{\prime}(t) & =S(t) b(S(t))-g(I(t)) S(t), & & t \neq(n+l-1) T, t \neq n T ; \\
I^{\prime}(t) & =g(I(t)) S(t)-w I(t), & & t \neq(n+l-1) T, t \neq n T ; \\
\Delta S(t) & =-\delta_{1} S(t), & & t=(n+l-1) T ; \\
\Delta I(t) & =-\delta_{I} I(t), & & t=(n+l-1) T ; \\
\Delta S(t) & =0, & & t=n T ; \\
\Delta I(t) & =\mu, & & t=n T .
\end{aligned}\right.
$$

For the sake of comparison and for further reference we shall indicate here the controllability results obtained by Georgescu and Moroşanu ${ }^{38}$ and the bifurcation results deduced by Georgescu, Zhang and Chen. ${ }^{70}$

Let us denote by $I_{w}^{*}$ the periodic solution of the following subsystem of (2.2), which describes the dynamics of the susceptible pest-free state,

$$
\left\{\begin{aligned}
I^{\prime}(t) & =-w I(t), & & t \neq n T,(n+l-1) T \\
\Delta I(t) & =-\delta_{I} I(t), & & t=(n+l-1) T \\
\Delta I(t) & =\mu, & & t=n T .
\end{aligned}\right.
$$

It is seen that $I_{w}^{*}$ is given by

$$
I_{w}^{*}= \begin{cases}\frac{\mu}{1-e^{-w T}\left(1-\delta_{I}\right)} e^{-w(t-n T)}, & t \in(n T,(n+l) T] \\ \frac{\mu\left(1-\delta_{I}\right)}{1-e^{-w T}\left(1-\delta_{I}\right)} e^{-w(t-n T)}, & t \in((n+l) T,(n+1) T] .\end{cases}
$$

Also, it has been proved by Georgescu and Moroşanu ${ }^{38}$ that the following dichotomy result holds.

Theorem 2.1. (Georgescu and Moroşanu ${ }^{38}$ ) The following statements hold.

(1) The susceptible pest-eradication periodic solution $\left(0, I_{w}^{*}\right)$ of $(2.2)$ is globally asymptotically stable provided that

$$
\int_{0}^{T} g\left(I_{w}^{*}(s)\right) d s-\ln \left(1-\delta_{1}\right)>r T .
$$


(2) The susceptible pest-eradication periodic solution $\left(0, I_{w}^{*}\right)$ of $(2.2)$ is unstable provided that

$$
\int_{0}^{T} g\left(I_{w}^{*}(s)\right) d s-\ln \left(1-\delta_{1}\right)<r T .
$$

In this case, (2.2) is also permanent (both susceptible and infective populations survive in the long-term).

In the limit case, i.e., the case in which

$$
\int_{0}^{T} g\left(I_{w}^{*}(s)\right) d s-\ln \left(1-\delta_{1}\right)=r T,
$$

it is seen that the susceptible pest-eradication periodic solution $\left(0, I_{w}^{*}\right)$ is stable, but not necessarily asymptotically stable, and a supercritical bifurcation occurs, as established in the following result obtained by Georgescu, Zhang and Chen. ${ }^{70}$

Theorem 2.2. (Georgescu, Zhang and $\mathrm{Chen}^{70}$ ) A supercritical bifurcation occurs if (2.7) is crossed, in the sense that a stable positive periodic solution bifurcates from the susceptible pest-eradication periodic solution.

Indeed, numerical experiments (see Fig. 4) indicate that further period-doubling bifurcations occur beyond this crossing, in the unstable parameter regime.

Note that the conditions (2.5)-(2.7) have an immediate biological significance, as they represent balance conditions near the susceptible pest-eradication periodic solution $\left(0, I_{w}^{*}\right)$. Specifically, if $(S, I)$ approaches $\left(0, I_{w}^{*}\right)$, then the integral $\int_{0}^{T} g\left(I_{w}^{*}(t)\right) d t$ approximate the (normalized, per-susceptible) movement of susceptible pests to the infective class in a given period, $r T$ approximates the (normalized, per-susceptible) amount of susceptible pests which are born in a given period, while $\ln \left(1-\delta_{1}\right)$ is a correction term which represents the (normalized, per-susceptible) loss of susceptible pests due to pesticide spraying.

It is also to be seen that the stability of $\left(0, I_{w}^{*}\right)$ cannot be characterized by a basic reproduction number defined in the classical sense, although $R_{S}=$ $\frac{\int_{0}^{T} g\left(I_{w}^{*}(t)\right) d t-\ln \left(1-\delta_{1}\right)}{r T}$ is a threshold parameter for the stability of $(2.2)$, since the basic reproduction number is usually employed to characterize the dynamics of the system near the infective-free state, in a system with no impulsive perturbations. Of concern here is the extinction or nonextinction of susceptible pests, rather than of infective pests, since due to the periodic release of infective pests, which occurs at $t=n T$, the infective pest population never dies out. Consequently, we may not expect the existence of a basic reproduction number in the classical sense for the two-patched system. An additional difficulty consists in the fact that the system approaches a periodic solution, rather than a stationary state. In this regard, the formula for the basic reproduction number $R_{0}$ in a constant environment has been generalized to the case of a periodic environment by Bacaër and Ouifki, ${ }^{71}$ Bacaër ${ }^{72}$ and Wang and Zhao; ${ }^{73}$ however, these models do not account for the effect of impulsive perturbations. 


\section{Georgescu, Dimitriu \& Sinclair}

It is to be noted that our model can be used to describe some situations which are apparently not covered by (2.1). In this sense, suppose that the pests in the first patch grow according to a logistic growth rate with intrinsic birth rate $r$ and carrying capacity $K$. The infective pests in the first patch do contribute towards the carrying capacity of the environment and consequently the first equation in our model is replaced by,

$$
S_{1}^{\prime}(t)=r S_{1}(t)\left(1-\frac{S_{1}(t)+\alpha I_{1}(t)}{K}\right)-g\left(I_{1}(t)\right) S_{1}(t)+d_{21} S_{2}(t)-d_{12} S_{1}(t),
$$

where $\alpha$ is a constant which characterizes the fact that susceptible and infective pests have different capabilities to damage the environment. Then (2.8) can be rearranged as,

$$
S_{1}^{\prime}(t)=r S_{1}(t)\left(1-\frac{S_{1}(t)}{K}\right)-\left(g\left(I_{1}(t)\right)+\frac{\alpha r I_{1}(t)}{K}\right) S_{1}(t)+d_{21} S_{2}(t)-d_{12} S_{1}(t),
$$

which fits our framework for the new function $\tilde{g}\left(I_{1}\right)=g\left(I_{1}\right)+\alpha r \frac{I_{1}}{K}$, although this time the limiting size of the infective pest population is also of concern. Specifically, the global stability of the susceptible pest-eradication periodic solution would not suffice, another requirement being that the average endemicity level be lower than a certain value which is determined knowing the value of the EIL. Also, if the infective pests do reproduce, but with purely vertical transmission (i.e., any offspring of an infective is an infective) then the third equation of the model is replaced by,

$$
I_{1}^{\prime}=\beta I_{1}(t)+g\left(I_{1}(t)\right) S_{1}(t)-w_{1} I_{1}(t)+D_{12} I_{1}(t)-D_{21} I_{2}(t),
$$

where $\beta$ denotes the (constant) birth rate for the infective compartment, then this equation can be rearranged as,

$$
I_{1}^{\prime}(t)=g\left(I_{1}(t)\right) S_{1}(t)-\left(w_{1}-\beta\right) I_{1}(t)+D_{12} I_{1}(t)-D_{21} I_{2}(t),
$$

which again fits our framework provided that $\beta<w_{1}$.

\section{Preliminaries}

As in the single-patch case, the pulsed supply of infective pests which occurs at $t=n T$ and the periodic pesticide spraying which occurs at $t=(n+l-1) T$ should be regarded as periodic forcings which are expected to ensure some stability properties for a semitrivial (susceptible pest-free) periodic solution $E^{*}$, whose existence is yet to be determined. In the following, we shall introduce several notions of Floquet theory for linear differential systems with periodic coefficients and of time-dependent matrix exponentials which are necessary in order to discuss such stability properties.

Let us consider the homogeneous, first order system of $n$ linear differential equations with periodic coefficients and periodic impulsive perturbations

$$
\begin{cases}x^{\prime}(t)=A(t) x, & t \neq \tau_{k}, \quad t \in \mathbb{R} \\ \Delta x=B_{k} x, & t=\tau_{k}, \quad \tau_{k}<\tau_{k+1}, \quad k \in \mathbb{Z}\end{cases}
$$


under the following hypotheses:

(H1) $A(\cdot) \in P C\left(\mathbb{R}, M_{n}(\mathbb{R})\right)$ and there is $T>0$ such that $A(t+T)=A(t)$ for all $t \geq 0$.

(H2) $B_{k} \in M_{n}(\mathbb{R}), \operatorname{det}\left(I_{n}+B_{k}\right) \neq 0$ for $k \in \mathbb{Z}$.

(H3) There is $q \in \mathbb{N}^{*}$ such that $B_{k+q}=B_{k}, \tau_{k+q}=\tau_{k}+T$ for $k \in \mathbb{Z}$.

In the above hypotheses, $P C\left(\mathbb{R}_{+}, \mathbb{R}\right)\left(P C^{1}\left(\mathbb{R}_{+}, \mathbb{R}\right)\right)$ denotes the class of real piecewise continuous (real piecewise continuously differentiable) functions defined on $[0, \infty)$.

Now let $\Phi(t)$ be a fundamental matrix of (3.1). Then there is a unique nonsingular matrix $M \in M_{n}(\mathbb{R})$ such that $\Phi(t+T)=\Phi(t) M$ for all $t \geq 0$, which is called the monodromy matrix of (3.1) associated to $\Phi$. Obviously, the monodromy matrix $M$ of (3.1) is not uniquely determined, as it depends on $\Phi$, but all monodromy matrices, being similar, have the same eigenvalues, called the Floquet multipliers of (3.1). These eigenvalues determine whether $\Phi(t)$ "shrinks" or "expands" with time, in the sense that the following stability result holds:

Lemma 3.1. (Bainov and Simeonov $\left.{ }^{18}\right)$ Suppose that conditions (H1)-(H3) hold. Then,

(1) The system (3.1) is stable if and only if all Floquet multipliers $\lambda_{k}, 1 \leq k \leq n$, satisfy $\left|\lambda_{k}\right| \leq 1$ and if $\left|\lambda_{k}\right|=1$, then to $\lambda_{k}$ there corresponds a simple elementary divisor.

(2) The system (3.1) is asymptotically stable if and only if all Floquet multipliers $\lambda_{k}, 1 \leq k \leq n$, satisfy $\left|\lambda_{k}\right|<1$.

(3) The system (3.1) is unstable if there is a Floquet multiplier $\lambda_{k}$ such that $\left|\lambda_{k}\right|>1$.

Here, by elementary divisors of a square matrix we understand the characteristic polynomials of its Jordan blocks. In order to use the above result, one often needs to compute explicitly a monodromy matrix, which is feasible provided that the fundamental matrix of the unperturbed system consisting of the first part of (3.1) is written as a matrix exponential. Several remarks in this direction are given in Appendix A.

\section{The Dynamics of the Susceptible Pest-Eradication Periodic Solution}

Here, we shall consider that the following relation between death rates and dispersal rates of infective pests holds:

$$
w_{2}-w_{1}=2\left(D_{12}-D_{21}\right) .
$$


Note that this equality, motivated by the need of constructing the exponential of a certain time-dependent matrix, the technical reason for which will be detailed below, is satisfied, for instance, if $w_{1}=w_{2}=w$ and $D_{12}=D_{21}=D$ (i.e., if the mortality rate of infective pests is the same in each patch and their dispersal is proportional to the difference in their respective population sizes). We do provide a numerical example for which this relation does not hold (Fig. 5).

Let us consider the following subsystem of (2.1), which models the dynamics of the susceptible pest-eradication state:

$$
\left\{\begin{aligned}
I_{1}^{\prime}(t) & =-\left(w_{1}+D_{12}\right) I_{1}(t)+D_{21} I_{2}(t), & & t \neq(n+l-1) T, \quad t \neq n T \\
I_{2}^{\prime}(t) & =D_{12} I_{1}(t)-\left(w_{2}+D_{21}\right) I_{2}(t), & & t \neq(n+l-1) T, \quad t \neq n T \\
\Delta I_{i}(t) & =-\delta_{I} I_{i}(t), & & t=(n+l-1) T, \quad i=1,2 \\
\Delta I_{i}(t) & =\mu, & & t=n T, \quad i=1,2 . \\
I_{i}(0+) & =I_{i}^{0} & & i=1,2 .
\end{aligned}\right.
$$

The system which consists of the first four equations of (4.2) has a globally attracting periodic solution $\left(I^{*}, I^{*}\right)^{t}$, as shown in the following lemma. Here, we shall denote by $(x, y)^{t}$ the transpose of $(x, y)$.

Lemma 4.1. There is a T-periodic solution $\left(I^{*}, I^{*}\right)^{t}$ of the first four equations of (4.2), such that,

$$
\lim _{t \rightarrow \infty}\left(\left|I_{1}(t)-I^{*}(t)\right|+\left|I_{2}(t)-I^{*}(t)\right|\right)=0
$$

for all solutions $\left(I_{1}, I_{2}\right)^{t}$ of $(4.2)$.

The proof of Lemma 4.1 is given in Appendix B.

\section{The Stability Result}

In this section, we shall establish the well-posedness of the system (2.1) in a mathematical and biological sense and discuss the stability of the susceptible pest-eradication periodic solution $E^{*}=\left(0,0, I^{*}, I^{*}\right)$ by using the method of small amplitude perturbations. First of all, it is easy to see that (2.1) has a unique solution for all sets of initial data. The proofs of the following lemmas, which establish the well-posedness of the system (2.1) in a biological sense, are immediate.

Lemma 5.1. The set $(0, \infty)^{4}$ is an invariant region for the system $(2.1)$.

Thus, numbers of both susceptible and infective pests remain non-negative for all time and no population explosion, in which pest numbers would become infinite, can occur. 
Lemma 5.2. There is $M>0$ such that $S_{i}(t) \leq M, I_{i}(t) \leq M$ for $t \geq 0, i=1,2$.

We now begin to discuss the stability of $E^{*}=\left(0,0, I^{*}, I^{*}\right)$. To this purpose, let us denote

$$
\left\{\begin{array}{l}
S_{1}(t)=u_{1}(t) ; \\
S_{2}(t)=u_{2}(t) ; \\
I_{1}(t)=v_{1}(t)+I^{*}(t) ; \\
I_{2}(t)=v_{2}(t)+I^{*}(t),
\end{array}\right.
$$

where, $u_{i}, v_{i}, i=1,2$ are understood to be small amplitude perturbations. By substituting (5.1) into (2.1), one obtains,

$$
\left\{\begin{array}{l}
u_{1}^{\prime}(t)=u_{1}(t) b_{1}\left(u_{1}(t)\right)-g\left(v_{1}(t)+I^{*}(t)\right) u_{1}(t)+d_{21} u_{2}(t)-d_{12} u_{1}(t) ; \\
u_{2}^{\prime}(t)=u_{2}(t) b_{2}\left(u_{2}(t)\right)-g\left(v_{2}(t)+I^{*}(t)\right) u_{2}(t)+d_{12} u_{1}(t)-d_{21} u_{2}(t) ; \\
\left.v_{1}^{\prime}(t)=g\left(v_{1}(t)\right)+I^{*}(t)\right) u_{1}(t)-w_{1} v_{1}(t)-D_{12} v_{1}(t)+D_{21} v_{2}(t) \\
\left.v_{2}^{\prime}(t)=g\left(v_{2}(t)\right)+I^{*}(t)\right) u_{2}(t)-w_{2} v_{2}(t)+D_{12} v_{1}(t)-D_{21} v_{2}(t)
\end{array} .\right.
$$

The linearization of (5.2) around $(0,0,0,0)^{t}$ reads as,

$$
\left\{\begin{array}{l}
u_{1}^{\prime}(t)=r_{1} u_{1}(t)-g\left(I^{*}(t)\right) u_{1}(t)+d_{21} u_{2}(t)-d_{12} u_{1}(t) \\
u_{2}^{\prime}(t)=r_{2} u_{2}(t)-g\left(I^{*}(t)\right) u_{2}(t)+d_{12} u_{1}(t)-d_{21} u_{2}(t) \\
v_{1}^{\prime}(t)=g\left(I^{*}(t)\right) u_{1}(t)-w v_{1}(t)-D_{12} v_{1}(t)+D_{21} v_{2}(t) \\
v_{2}^{\prime}(t)=g\left(I^{*}(t)\right) u_{2}(t)-w v_{2}(t)+D_{12} v_{1}(t)-D_{21} v_{2}(t)
\end{array}\right.
$$

while the linearization of the jump conditions at $(n+l-1) T$ is,

$$
\left\{\begin{array}{l}
\Delta u_{1}=-\delta_{1} u_{1}(t), \quad t=(n+l-1) T \\
\Delta u_{2}=-\delta_{2} u_{2}(t) \\
\Delta v_{1}=-\delta_{I} v_{1}(t) \\
\Delta v_{2}=-\delta_{I} v_{2}(t)
\end{array}\right.
$$

and the linearization of the jump conditions at $n T$ is,

$$
\Delta u_{1}=\Delta u_{2}=\Delta v_{1}=\Delta v_{2}=0, \quad t=n T .
$$


Let us use the following block writing of the (time-dependent) matrix $\mathcal{M}$ of (5.3)

$$
\begin{aligned}
\mathcal{M} & =\left(\begin{array}{cccc}
\left(r_{1}-d_{12}\right)-g\left(I^{*}(t)\right) & d_{21} & 0 & 0 \\
d_{12} & \left(r_{2}-d_{21}\right)-g\left(I^{*}(t)\right) & 0 & 0 \\
g\left(I^{*}(t)\right) & 0 & -\left(w_{1}+d_{12}\right) & d_{21} \\
0 & g\left(I^{*}(t)\right) & d_{12} & -\left(w_{2}+d_{21}\right)
\end{array}\right) \\
& =\left(\begin{array}{cc}
A(t) & O_{2} \\
g\left(I^{*}(t)\right) I_{2} & -C
\end{array}\right) .
\end{aligned}
$$

It is then seen that the following commutation condition holds,

$$
A(t)\left(\int_{0}^{t} A(s) d s\right)=\left(\int_{0}^{t} A(s) d s\right) A(t), \quad \forall t \geq 0 .
$$

The proof of this fact is given in Appendix C, together with a motivation for using the same nonlinear force of infection $g$ and the same proportional loss of infective pests $\delta_{I}$ in each patch and also for using condition (4.1).

The fundamental matrix $\Phi$ of the linearized system (5.3) is then given by,

$$
\Phi(t)=\left(\begin{array}{cc}
\exp \left(\int_{0}^{t} A(s) d s\right) & O_{2} \\
\int_{0}^{t} \exp ((t-s) C) g\left(I^{*}(s)\right) \exp \left(\int_{0}^{s} A(\tau) d \tau\right) d s & \exp (t C)
\end{array}\right)
$$

to which there corresponds the following monodromy matrix:

$$
M=\left(\begin{array}{cc}
\left(\begin{array}{cc}
1-\delta_{1} & 0 \\
0 & 1-\delta_{2}
\end{array}\right) \exp \left(\int_{0}^{T} A(s) d s\right) & O_{2} \\
\left(1-\delta_{I}\right) \int_{0}^{T} \exp ((T-s) C) g\left(I^{*}(s)\right) \exp \left(\int_{0}^{s} A(\tau) d \tau\right) d s & \left(1-\delta_{I}\right) \exp (C T)
\end{array}\right) .
$$

Since the eigenvalues of $\left(1-\delta_{I}\right) \exp (T C)$ are $\left(1-\delta_{I}\right) e^{\lambda_{1} T},\left(1-\delta_{I}\right) e^{\lambda_{2} T} \in(0,1)$, it follows that the stability of the susceptible pest-eradication periodic solution is determined by the eigenvalues of the matrix,

$$
M_{1}=\left(\begin{array}{cc}
1-\delta_{1} & 0 \\
0 & 1-\delta_{2}
\end{array}\right) \exp \left(\int_{0}^{T} A(s) d s\right)
$$

called in what follows the reduced monodromy matrix. We first prove that these eigenvalues are actually positive real numbers. To this purpose, we compute $\exp \left(\int_{0}^{T} A(t) d t\right)$. It is seen that,

$$
\int_{0}^{T} A(t) d t=\left(\begin{array}{cc}
\alpha_{1} & d_{21} T \\
d_{12} T & \alpha_{2}
\end{array}\right)
$$


where

$$
\begin{aligned}
& \alpha_{1}=\int_{0}^{T} a_{1}(t) d t=\left(r_{1}-d_{12}\right) T-\int_{0}^{T} g\left(I^{*}(t)\right) d t \\
& \alpha_{2}=\int_{0}^{T} a_{2}(t) d t=\left(r_{2}-d_{21}\right) T-\int_{0}^{T} g\left(I^{*}(t)\right) d t .
\end{aligned}
$$

The eigenvalues of $\int_{0}^{T} A(t) d t$ are then,

$$
\begin{aligned}
& \xi_{1}=\frac{1}{2}\left(\alpha_{1}+\alpha_{2}+\sqrt{\left(\alpha_{1}-\alpha_{2}\right)^{2}+4 d_{12} d_{21} T^{2}}\right) \\
& \xi_{2}=\frac{1}{2}\left(\alpha_{1}+\alpha_{2}-\sqrt{\left(\alpha_{1}-\alpha_{2}\right)^{2}+4 d_{12} d_{21} T^{2}}\right)
\end{aligned}
$$

the two associated eigenvectors being,

$$
v_{1}=\left(\begin{array}{c}
d_{21} T \\
\xi_{1}-\alpha_{1}
\end{array}\right), \quad v_{2}=\left(\begin{array}{c}
d_{21} T \\
\xi_{2}-\alpha_{1}
\end{array}\right)
$$

From (5.7), we have,

$$
\xi_{1} \geq \max \left(\alpha_{1}, \alpha_{2}\right), \quad \xi_{2} \leq \min \left(\alpha_{1}, \alpha_{2}\right) .
$$

Consequently, since

$$
\int_{0}^{T} A(s) d s=\left(\begin{array}{cc}
d_{21} T & d_{21} T \\
\xi_{1}-\alpha_{1} & \xi_{2}-\alpha_{1}
\end{array}\right)\left(\begin{array}{cc}
\xi_{1} & 0 \\
0 & \xi_{2}
\end{array}\right)\left(\begin{array}{cc}
d_{21} T & d_{21} T \\
\xi_{1}-\alpha_{1} & \xi_{2}-\alpha_{1}
\end{array}\right)^{-1}
$$

it follows that,

$$
\exp \left(\int_{0}^{T} A(s) d s\right)=\left(\begin{array}{cc}
d_{21} T & d_{21} T \\
\xi_{1}-\alpha_{1} & \xi_{2}-\alpha_{1}
\end{array}\right)\left(\begin{array}{cc}
e^{\xi_{1}} & 0 \\
0 & e^{\xi_{2}}
\end{array}\right)\left(\begin{array}{cc}
d_{21} T & d_{21} T \\
\xi_{1}-\alpha_{1} & \xi_{2}-\alpha_{1}
\end{array}\right)^{-1}
$$

and the reduced monodromy matrix $M_{1}$ is given by,

$$
M_{1}=\left(\begin{array}{cc}
\left(1-\delta_{1}\right) \frac{\left(\xi_{1}-\alpha_{1}\right) e^{\xi_{2}}-\left(\xi_{2}-\alpha_{1}\right) e^{\xi_{1}}}{\xi_{1}-\xi_{2}} & \left(1-\delta_{1}\right) \frac{d_{21} T\left(e^{\xi_{1}}-e^{\xi_{2}}\right)}{\xi_{1}-\xi_{2}} \\
\left(1-\delta_{2}\right) \frac{\left(\xi_{1}-\alpha_{1}\right)\left(\xi_{2}-\alpha_{1}\right)\left(e^{\xi_{2}}-e^{\xi_{1}}\right)}{d_{21} T\left(\xi_{1}-\xi_{2}\right)} & \left(1-\delta_{2}\right) \frac{\left(\xi_{1}-\alpha_{1}\right) e^{\xi_{1}}-\left(\xi_{2}-\alpha_{1}\right) e^{\xi_{2}}}{\xi_{1}-\xi_{2}}
\end{array}\right) .
$$

Now

$$
\begin{aligned}
& \mu_{1}=\frac{\operatorname{Tr} M_{1}+\sqrt{\left(\operatorname{Tr} M_{1}\right)^{2}-4 \operatorname{det} M_{1}}}{2}, \\
& \mu_{2}=\frac{\operatorname{Tr} M_{1}-\sqrt{\left(\operatorname{Tr} M_{1}\right)^{2}-4 \operatorname{det} M_{1}}}{2},
\end{aligned}
$$


where

$$
\begin{aligned}
\operatorname{Tr} M_{1}= & \left(1-\delta_{1}\right) \frac{\left(\xi_{1}-\alpha_{1}\right) e^{\xi_{2}}-\left(\xi_{2}-\alpha_{1}\right) e^{\xi_{1}}}{\xi_{1}-\xi_{2}} \\
& +\left(1-\delta_{2}\right) \frac{\left(\xi_{1}-\alpha_{1}\right) e^{\xi_{1}}-\left(\xi_{2}-\alpha_{1}\right) e^{\xi_{2}}}{\xi_{1}-\xi_{2}} \\
\operatorname{det} M_{1}= & \left(1-\delta_{1}\right)\left(1-\delta_{2}\right) e^{\xi_{1}+\xi_{2}}
\end{aligned}
$$

Since $\xi_{1}-\alpha_{1} \geq 0, \xi_{2}-\alpha_{1} \leq 0, \xi_{1}-\xi_{2} \geq 0$, it follows that $\operatorname{Tr} M_{1} \geq 0$, $\operatorname{det} M_{1} \geq 0$. Consequently

$$
\begin{aligned}
\left(\operatorname{Tr} M_{1}\right)^{2}-4 \operatorname{det} M_{1}= & {\left[\left(1-\delta_{1}\right) \frac{\left(\xi_{1}-\alpha_{1}\right) e^{\xi_{2}}-\left(\xi_{2}-\alpha_{1}\right) e^{\xi_{1}}}{\xi_{1}-\xi_{2}}\right.} \\
& \left.\quad-\left(1-\delta_{2}\right) \frac{\left(\xi_{1}-\alpha_{1}\right) e^{\xi_{1}}-\left(\xi_{2}-\alpha_{1}\right) e^{\xi_{2}}}{\xi_{1}-\xi_{2}}\right]^{2} \\
& +4 \frac{\left(1-\delta_{1}\right)\left(1-\delta_{2}\right)}{\left(\xi_{1}-\xi_{2}\right)^{2}}\left(-\left(\xi_{1}-\alpha_{1}\right)\left(\xi_{2}-\alpha_{1}\right)\right)\left(e^{\xi_{1}}-e^{\xi_{2}}\right)^{2} \\
\geq & 0 .
\end{aligned}
$$

It then follows from (5.10), (5.11) and (5.12) that $0<\mu_{2}<\mu_{1}$ and the susceptible pest-eradication periodic solution is stable provided that,

$$
\operatorname{Tr} M_{1}+\sqrt{\left(\operatorname{Tr} M_{1}\right)^{2}-4 \operatorname{det} M_{1}} \leq 2,
$$

or unstable provided that the reverse inequality holds. Since the interpretation of the stability condition (5.13) is somewhat difficult, we shall concentrate on several particular cases. Namely, we shall discuss the case in which there is no dispersal of pests and the case in which the chemical control is equally efficient in both patches, the last one being further specialized.

\subsection{No dispersal $\left(d_{12}=d_{21}=D_{12}=D_{21}=0\right)$}

In this case, necessarily $w_{1}=w_{2}=w$, due to (4.1), and consequently

$$
\mathcal{M}=\left(\begin{array}{cccc}
r_{1}-g\left(I^{*}(t)\right) & 0 & 0 & 0 \\
0 & r_{2}-g\left(I^{*}(t)\right) & 0 & 0 \\
g\left(I^{*}(t)\right) & 0 & -w & 0 \\
0 & g\left(I^{*}(t)\right) & 0 & -w
\end{array}\right),
$$

which implies that the monodromy matrix is,

$$
M=\left(\begin{array}{cccc}
\left(1-\delta_{1}\right) e^{r_{1} T-\int_{0}^{T} g\left(I^{*}(t)\right) d t} & 0 & 0 & 0 \\
0 & \left(1-\delta_{2}\right) e^{r_{2} T-\int_{0}^{T} g\left(I^{*}(t)\right) d t} & 0 & 0 \\
m_{31} & 0 & e^{-w T} & 0 \\
0 & m_{42} & 0 & e^{-w T}
\end{array}\right) .
$$


where

$$
\begin{aligned}
& m_{31}=\left(1-\delta_{I}\right) \int_{0}^{T} e^{-w(T-s) g\left(I^{*}(s)\right) e^{r_{1} s}-\int_{0}^{s} g\left(I^{*}(\tau)\right) d \tau} d s \\
& m_{42}=\left(1-\delta_{I}\right) \int_{0}^{T} e^{-w(T-s) g\left(I^{*}(s)\right) e^{r_{2} s}-\int_{0}^{s} g\left(I^{*}(\tau)\right) d \tau} d s
\end{aligned}
$$

In this case, the stability condition becomes

$$
\max \left(\left(1-\delta_{1}\right) e^{r_{1} T-\int_{0}^{T} g\left(I^{*}(t)\right) d t},\left(1-\delta_{2}\right) e^{r_{2} T-\int_{0}^{T} g\left(I^{*}(t)\right) d t}\right) \leq 1,
$$

i.e., $E^{*}$ is stable if $\left(0, I^{*}\right)$ is stable in each patch in isolation, as seen from $(2.5)-(2.7)$.

\subsection{Equal efficiency of chemical control in both patches

$$
\left(\delta_{1}=\delta_{2}=\delta\right)
$$

In this case, it is seen from (5.10) that,

$$
\operatorname{Tr} M_{1}=(1-\delta)\left(e^{\xi_{1}}+e^{\xi_{2}}\right), \quad \operatorname{det} M_{1}=(1-\delta)^{2} e^{\xi_{1}+\xi_{2}}
$$

and consequently, from (5.9),

$\mu_{1}=(1-\delta) \max \left(e^{\xi_{1}}, e^{\xi_{2}}\right)=(1-\delta) e^{\xi_{1}}, \quad \mu_{2}=(1-\delta) \min \left(e^{\xi_{1}}, e^{\xi_{2}}\right)=(1-\delta) e^{\xi_{2}}$.

It follows that the sufficient condition for stability is $\ln (1-\delta)+\xi_{1} \leq 0$, i.e.,

$$
E\left(r_{1}, r_{2}, d_{12}, d_{21}, w_{1}, w_{2}, g, T\right) \leq 0,
$$

where

$$
\begin{aligned}
& E\left(r_{1}, r_{2}, d_{12}, d_{21}, w_{1}, w_{2}, g, T\right) \\
& =2 \ln (1-\delta)+\left(r_{1}-d_{12}\right) T+\left(r_{2}-d_{21}\right) T-2 \int_{0}^{T} g\left(I^{*}(t)\right) d t \\
& \quad+\sqrt{\left(\left(r_{1}-d_{12}\right) T-\left(r_{2}-d_{21}\right) T\right)^{2}+4 d_{12} d_{21} T^{2}}
\end{aligned}
$$

Note that

$$
E\left(r, r, d_{12}, d_{21}, w_{1}, w_{2}, g, T\right)=2 \ln (1-\delta)+2 r T-2 \int_{0}^{T} g\left(I^{*}(t)\right) d t,
$$

so the dispersal of susceptible pests has no influence upon the stability of $E^{*}$, provided that the growth rates $r_{1}, r_{2}$ are the same in each patch.

We further specialize our results for this situation. We first consider the case of a linear force of infection $g$, which allows us to compute the integral term which appears in (5.20) explicitly.

\subsection{1. $\delta_{1}=\delta_{2}=\delta, g(x)=a x$}

In this case, $\int_{0}^{T} g\left(I^{*}(t)\right) d t=\frac{2 a \mu}{w_{1}+w_{2}}$. The stability conditions for each patch are $\ln (1-\delta)+r_{1} T-\frac{a \mu}{w_{1}} \leq 0$ and $\ln (1-\delta)+r_{2} T-\frac{a \mu}{w_{2}} \leq 0$, respectively. 
Further, for $d_{12}=d_{21}=0$ (i.e., no dispersion of susceptible pests between patches), it is seen that

$$
E\left(r_{1}, r_{2}, 0,0, w_{1}, w_{2}, a, T\right)=2 \ln (1-\delta)+r_{1} T+r_{2} T-\frac{4 a \mu}{w_{1}+w_{2}}+\left|r_{1} T-r_{2} T\right| .
$$

Now let us consider some special cases:

(a) Both patches are unstable without dispersion.

Then

$$
\begin{aligned}
E\left(r_{1}, r_{2}, 0,0, w_{1}, w_{2}, a, T\right)= & \ln (1-\delta)+\left(r_{1} T-\frac{a \mu}{w_{1}}\right)+\ln (1-\delta)+\left(r_{2} T-\frac{a \mu}{w_{2}}\right) \\
& +\left(\frac{a \mu}{w_{1}}+\frac{a \mu}{w_{2}}-\frac{4 a \mu}{w_{1}+w_{2}}\right)+\left|r_{1} T-r_{2} T\right|,
\end{aligned}
$$

i.e., $E^{*}$ is unstable regardless of the values of the dispersion rates $D_{12}$ and $D_{21}$.

(b) Both patches are stable without dispersion.

Then

$$
E\left(r_{1}, r_{2}, 0,0, w_{1}, w_{2}, a, T\right)=2 \max \left(\ln (1-\delta)+r_{1} T, \ln (1-\delta)+r_{2} T\right)-\frac{4 a \mu}{w_{1}+w_{2}} .
$$

Suppose $\max \left(r_{1} T, r_{2} T\right)=r_{1} T$. Then

$$
E\left(r_{1}, r_{2}, 0,0, w_{1}, w_{2}, a, T\right)=2\left(\ln (1-\delta)+r_{1} T-\frac{a \mu}{w_{1}}\right)+2 \frac{a \mu\left(w_{2}-w_{1}\right)}{w_{1}\left(w_{1}+w_{2}\right)} .
$$

Consequently, the stability of $E^{*}$ is uncertain, as $E \leq 0$ for $w_{1} \geq w_{2}$, while $E$ might take both positive and negative values for $w_{1}<w_{2}$, depending on the values of $\ln (1-\delta)+r_{1} T-\frac{a \mu}{w_{1}}$. That is, the dispersion of infective pests has the potential to destabilize an otherwise stable system. Note that if $w_{1}=w_{2}$ (i.e., the system consists of two identical patches and necessarily $D_{12}=D_{21}=D$ ), then $E \leq 0$ and $E^{*}$ is stable regardless of the value of $D$.

(c) One patch is stable and one patch is unstable without dispersion. Again, by the same argument, the stability of $E^{*}$ is uncertain.

For $r_{1}=r_{2}=r$ (i.e., the birth rates in each patch are equal) it is seen that

$$
\begin{aligned}
E\left(r, r, 0,0, w_{1}, w_{2}, a, T\right)= & 2 \ln (1-\delta)+2 r T-\frac{4 a \mu}{w_{1}+w_{2}} \\
= & \left(\ln (1-\delta)+r T-\frac{a \mu}{w_{1}}\right)+\left(\ln (1-\delta)+r T-\frac{a \mu}{w_{2}}\right) \\
& +\left(\frac{a \mu}{w_{1}}+\frac{a \mu}{w_{2}}-\frac{4 a \mu}{w_{1}+w_{2}}\right) .
\end{aligned}
$$

By the same argument, $E^{*}$ is unstable if both patches are unstable without dispersion, while if both patches are stable without dispersion, then the stability of $E^{*}$ is uncertain. Note again that the dispersal of susceptible pests has no influence upon the stability of $E^{*}$. 
For $d_{12}=d_{21}=d$ (i.e., the dispersal rates of susceptible pests between patches are equal), it is seen that,

$$
\begin{aligned}
E\left(r_{1},\right. & \left.r_{2}, d, d, w_{1}, w_{2}, a, T\right) \\
= & 2 \ln (1-\delta)+r_{1} T+r_{2} T-2 d T-\frac{4 a \mu}{w_{1}+w_{2}}+\sqrt{\left(r_{1} T-r_{2} T\right)^{2}+4 d^{2} T^{2}} \\
= & \left(\ln (1-\delta)+r_{1} T-\frac{a \mu}{w_{1}}\right)+\left(\ln (1-\delta)+r_{2} T-\frac{a \mu}{w_{2}}\right) \\
& +\left(\frac{a \mu}{w_{1}}+\frac{a \mu}{w_{2}}-\frac{4 a \mu}{w_{1}+w_{2}}\right)+\sqrt{\left(r_{1} T-r_{2} T\right)^{2}+4 d^{2} T^{2}}-2 d T .
\end{aligned}
$$

Again, $E^{*}$ is unstable if both patches are unstable without dispersion, while if both patches are stable without dispersion, then the stability of $E^{*}$ is uncertain. We continue our analysis with the case in which the mortality rates are equal in each patch.

5.2.2. $\delta_{1}=\delta_{2}=\delta, w_{1}=w_{2}=w$

In this case, necessarily $D_{12}=D_{21}=D$. Then

$$
\begin{aligned}
E\left(r_{1},\right. & \left.r_{2}, d_{12}, d_{21}, w_{1}, w_{2}, g, T\right) \\
= & 2 \ln (1-\delta)+\left(r_{1}-d_{12}\right) T+\left(r_{2}-d_{21}\right) T-2 \int_{0}^{T} g\left(I^{*}(s)\right) d s \\
& +\sqrt{\left[\left(\left(r_{1}-d_{12}\right) T-\left(r_{2}-d_{21}\right) T\right)\right]^{2}+4 d_{12} d_{21} T^{2}} .
\end{aligned}
$$

Note that in this case $\left(0, I^{*}\right)$ represents the susceptible pest-free periodic solution for each patch in isolation. It follows that,

$$
\begin{aligned}
& E\left(r_{1}, r_{2}, d_{12}, d_{21}, w, w, g, T\right) \\
& =\left(\ln (1-\delta)+r_{1} T-\int_{0}^{T} g\left(I^{*}(s)\right) d s\right)+\left(\ln (1-\delta)+r_{2} T-\int_{0}^{T} g\left(I^{*}(s)\right) d s\right) \\
& \quad+\sqrt{\left[\left(\left(r_{1}-d_{12}\right) T-\left(r_{2}-d_{21}\right) T\right)\right]^{2}+4 d_{12} d_{21} T^{2}}-\left(d_{12} T+d_{21} T\right) .
\end{aligned}
$$

(a) Both patches are stable without dispersal, i.e.,

$$
\ln (1-\delta)+r_{1} T-\int_{0}^{T} g\left(I^{*}(s)\right) d s \leq 0, \quad \ln (1-\delta)+r_{2} T-\int_{0}^{T} g\left(I^{*}(s)\right) d s \leq 0 .
$$

Then

$$
\begin{aligned}
& E\left(r_{1}, r_{2}, d_{12}, d_{21}, w, w, g, T\right) \\
& \quad \leq\left(\ln (1-\delta)+r_{1} T-\int_{0}^{T} g\left(I^{*}(s)\right) d s\right)+\left(\ln (1-\delta)+r_{2} T-\int_{0}^{T} g\left(I^{*}(s)\right) d s\right) \\
& \quad+\left|r_{1} T-r_{2} T\right|
\end{aligned}
$$




$$
\begin{aligned}
& \leq \max \left(\ln (1-\delta)+r_{1} T-\int_{0}^{T} g\left(I^{*}(s)\right) d s, \ln (1-\delta)+r_{2} T-\int_{0}^{T} g\left(I^{*}(s)\right) d s\right) \\
& \leq 0
\end{aligned}
$$

i.e., neither the dispersion of susceptible pests nor the dispersion of infective pests have the potential to destabilize the system.

(b) Both patches are unstable without dispersal, i.e.,

$$
\ln (1-\delta)+r_{1} T-\int_{0}^{T} g\left(I^{*}(s)\right) d s>0, \quad \ln (1-\delta)+r_{2} T-\int_{0}^{T} g\left(I^{*}(s)\right) d s>0
$$

Then

$$
\begin{aligned}
E\left(r_{1}, r_{2}, d_{12}, d_{21}, w, w, a, T\right) & \\
\geq & \left(\ln (1-\delta)+r_{1} T-\int_{0}^{T} g\left(I^{*}(s)\right) d s\right)+\left(\ln (1-\delta)+r_{2} T-\int_{0}^{T} g\left(I^{*}(s)\right) d s\right) \\
& \quad-\left|r_{1} T-r_{2} T\right| \\
\geq & \min \left(\ln (1-\delta)+r_{1} T-\int_{0}^{T} g\left(I^{*}(s)\right) d s, \ln (1-\delta)+r_{2} T-\int_{0}^{T} g\left(I^{*}(s)\right) d s\right) \\
\geq & 0,
\end{aligned}
$$

i.e., if both patches are unstable in isolation, then $E^{*}$ is unstable.

(c) One patch is stable and the other is unstable without dispersal. Suppose that the first patch is stable and the second is unstable, i.e., $\ln (1-\delta)+r_{1} T-$ $\int_{0}^{T} g\left(I^{*}(s)\right) d s \leq 0, \ln (1-\delta)+r_{2} T-\int_{0}^{T} g\left(I^{*}(s)\right) d s>0$.

If one of $d_{12}$ and $d_{21}$ is zero, then

$$
\begin{aligned}
& E\left(r_{1}, r_{2}, d_{12}, d_{21}, w, w, a, T\right) \\
& \quad=2 \max \left(\ln (1-\delta)+r_{1} T-\int_{0}^{T} g\left(I^{*}(s)\right) d s, \ln (1-\delta)+r_{2} T-\int_{0}^{T} g\left(I^{*}(s)\right) d s\right) .
\end{aligned}
$$

Consequently, if the dispersal rate of susceptible pests from the unstable patch to the stable patch is large enough and the dispersal rate of susceptible pests from the stable patch to the unstable patch is 0 , then $E^{*}$ is stable, i.e., the dispersal of susceptible pests from the unstable patch to the stable patch has the potential to stabilize the system. Also, if the dispersal rate of susceptible pests from the unstable patch to the stable patch is 0 , then $E^{*}$ is unstable, i.e., the dispersal of infective pests cannot stabilize the system. 


$$
\begin{aligned}
& \text { If } d_{12}=d_{21}=d \text {, then } \\
& \begin{aligned}
E\left(r_{1}, r_{2}, d, d, w, w, g, T\right) \\
=\left(\ln (1-\delta)+r_{1} T-\int_{0}^{T} g\left(I^{*}(s)\right) d s\right)+\left(\ln (1-\delta)+r_{2} T-\int_{0}^{T} g\left(I^{*}(s)\right) d s\right) \\
\quad+\frac{\left(r_{1}-r_{2}\right)^{2} T^{2}}{\sqrt{\left(r_{1}-r_{2}\right)^{2} T^{2}+4 d^{2} T^{2}}+2 d T}
\end{aligned}
\end{aligned}
$$

and consequently

$$
\begin{aligned}
\lim _{d \rightarrow \infty} E\left(r_{1}, r_{2}, d, d, w, w, g, T\right) \\
\quad=\left(\ln (1-\delta)+r_{1} T-\int_{0}^{T} g\left(I^{*}(s)\right) d s\right)+\left(\ln (1-\delta)+r_{2} T-\int_{0}^{T} g\left(I^{*}(s)\right) d s\right),
\end{aligned}
$$

i.e., large equal dispersal rates of susceptible pests might stabilize the system provided that,

$$
\left(\ln (1-\delta)+r_{1} T-\int_{0}^{T} g\left(I^{*}(s)\right) d s\right)+\left(\ln (1-\delta)+r_{2} T-\int_{0}^{T} g\left(I^{*}(s)\right) d s\right)<0
$$

or fail to stabilize it provided that the opposite inequality holds.

\section{Numerical Simulations}

Here, all units will be arbitrary.

To illustrate our mathematical findings, we proceed to further investigate the dynamics of the system (2.1) by using numerical simulations. To discuss the influence of dispersal upon the stability of the susceptible pest-eradication periodic solution $E^{*}$, let us first choose $g\left(I_{i}\right)=\frac{k I_{i}}{1+\alpha I_{i}}$ and $S_{i} b\left(S_{i}\right)=r_{i} S_{i}\left(1-\frac{S_{i}}{K}\right)^{2}$ for $i=1,2$, $T=1$ and $l=0.5$. We let $d_{12}$ vary over the interval $[0,1]$, with 20 division points. The solutions of the impulsively perturbed system (2.1) are integrated over 10 periods, with 110 time steps in each interval $[0, l T]$ and $[l T, T]$, using a fourth-order Runge-Kutta method, the variation of $S_{i}$ and $I_{i}, i=1,2$ with respect to $t$ and $d_{12}$ being illustrated in what follows by means of three-dimensional plots. On the $d_{12}$ and $t$ axes, the numbers refer to the indices of the division points, rather than to absolute values. Also, by a stable or unstable patch, respectively, we mean a patch in which, if considered in isolation, the corresponding susceptible pest-eradication periodic $\left(0, I^{*}\right)$ for that patch is stable or unstable, respectively.

For $d_{21}=0.01, D_{12}=0.8, D_{21}=0.7, \delta_{1}=0.2, \delta_{2}=0.4, \delta_{I}=0.5, w_{1}=0.1$, $w_{2}=0.3, \mu=0.03, r_{1}=0.99, r_{2}=0.01, k=1, K=8$, it is seen that the first patch is unstable, the second patch is stable and $E^{*}$ changes its stability for $d_{12}$ in $[0,1]$, going from unstable to stable at $d_{12}=0.75$. The behavior of the trajectory 

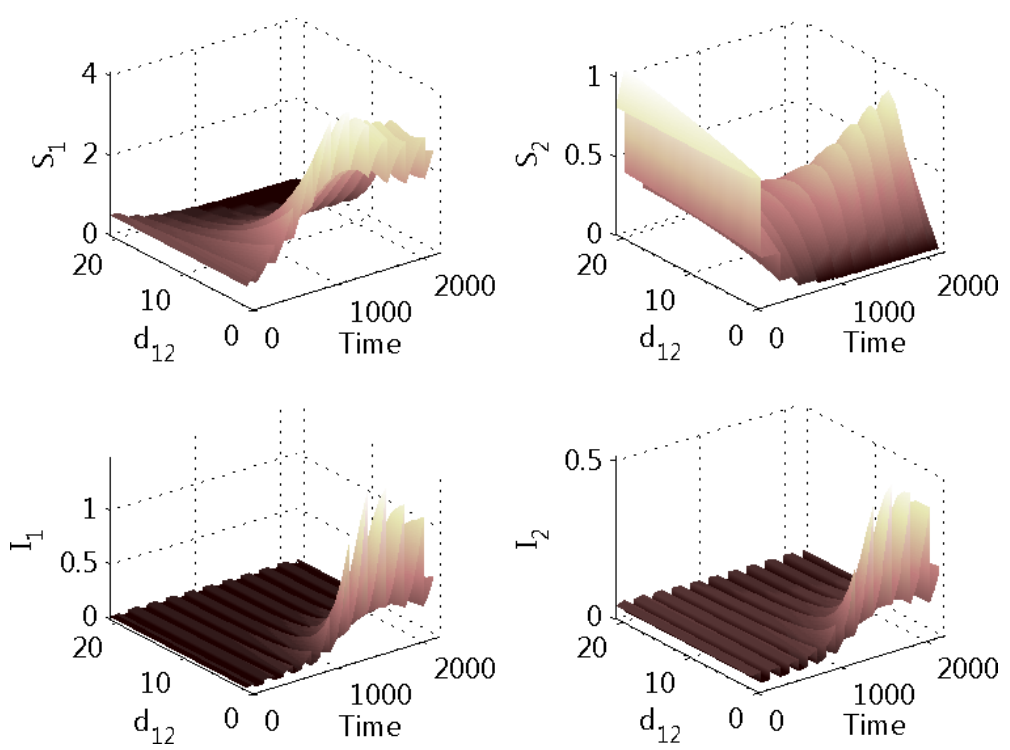

Fig. 1. $g\left(I_{i}\right)=\frac{k I_{i}}{1+\alpha I_{i}}$ and $S_{i} b\left(S_{i}\right)=r_{i} S_{i}\left(1-\frac{S_{i}}{K}\right)^{2}$ for $i=1,2, T=1, l=0.5, d_{21}=0.01$, $D_{12}=0.8, D_{21}=0.7, \delta_{1}=0.2, \delta_{2}=0.4, \delta_{I}=0.5, w_{1}=0.1, w_{2}=0.3, \mu=0.03, r_{1}=0.99$, $r_{2}=0.01, k=1, K=8$. An increase in the dispersal of susceptible pests from an unstable patch to a stable patch has the potential to contribute to the success of the pest control strategy.

starting with $S_{1}(0)=0.5, S_{2}(0)=0.8, I_{1}(0)=0.025, I_{2}(0)=0.04$ is depicted in Fig. 1. Since the dispersal of the susceptible pests from the second patch (the stable one) to the first patch (the unstable one) is weak, an increase in the dispersal of susceptible pests from the unstable patch to the stable patch has the potential to stabilize $E^{*}$ and therefore to contribute to the success of the pest control strategy. Note that the the gain of stability for $E^{*}$ occurs with an outburst in the size of the susceptible pest class in the stable patch.

For $d_{21}=0.95, D_{12}=0.85, D_{21}=0.7, \delta_{1}=0.4, \delta_{2}=0.1, \delta_{I}=0.5, w_{1}=0.1$, $w_{2}=0.3, \mu=0.03, r_{1}=0.95, r_{2}=0.9, k=1, K=8$, it is seen that both patches are unstable and $E^{*}$ is unstable for $d_{12}$ in $[0,1]$. Also, the value of the instabilitycausing eigenvalue $\mu_{1}$ is increasing with $d_{12}$. The behavior of the trajectory starting with $S_{1}(0)=0.5, S_{2}(0)=0.8, I_{1}(0)=0.025, I_{2}(0)=0.04$ is depicted in Fig. 2 . It is then seen that the sizes of the susceptible pest populations in both patches increase noticeably with the time, i.e., the dispersal of susceptible pest individuals from the first patch to the second patch has a negative impact upon the success of the pest control strategy, fact with can be attributed to the increase in the value of $\mu_{1}$.

For $d_{21}=0.25, D_{12}=0.9, D_{21}=0.8, \delta_{1}=0.25, \delta_{2}=0.2, \delta_{I}=0.9, w_{1}=0.1$, $w_{2}=0.3, \mu=0.03, r_{1}=0.25, r_{2}=0.5, k=2, K=8$, it is seen that the first patch is stable, the second patch is unstable and $E^{*}$ changes its stability for $d_{12}$ in $[0,1]$, going from stable to unstable at $d_{12}=0.1$. The behavior of the trajectory starting with $S_{1}(0)=0.5, S_{2}(0)=0.8, I_{1}(0)=0.025, I_{2}(0)=0.04$ is depicted in Fig. 3, 

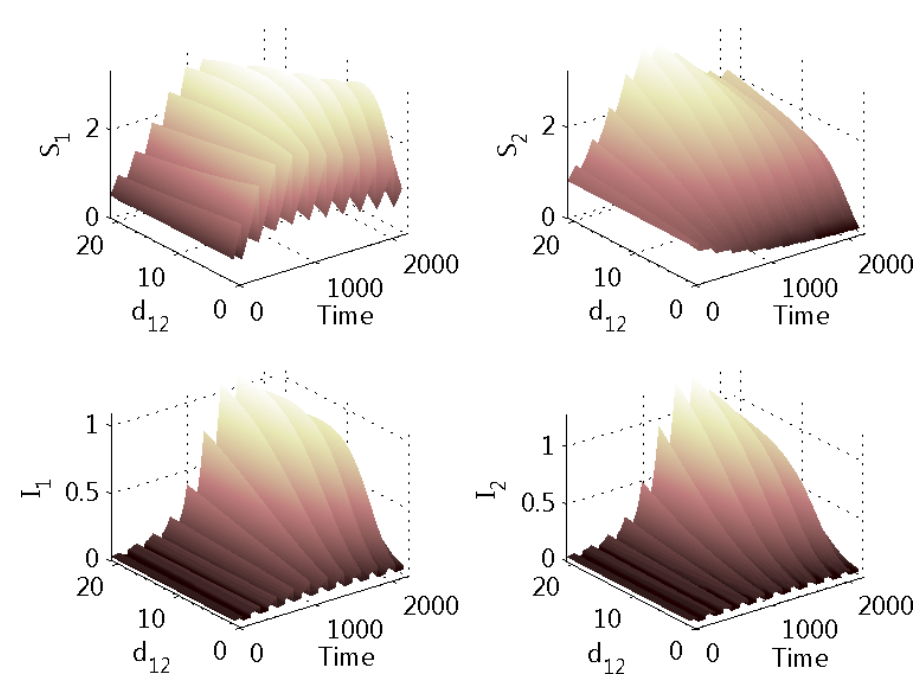

Fig. 2. $g\left(I_{i}\right)=\frac{k I_{i}}{1+\alpha I_{i}}$ and $S_{i} b\left(S_{i}\right)=r_{i} S_{i}\left(1-\frac{S_{i}}{K}\right)^{2}$ for $i=1,2, T=1, l=0.5, d_{21}=0.95$, $D_{12}=0.85, D_{21}=0.7, \delta_{1}=0.4, \delta_{2}=0.1, \delta_{I}=0.5, w_{1}=0.1, w_{2}=0.3, \mu=0.03, r_{1}=0.95$, $r_{2}=0.9, k=1, K=8$. The dispersal of susceptible pest individuals from patch $P_{1}$ to patch $P_{2}$ has a negative impact upon the success of the pest control strategy.
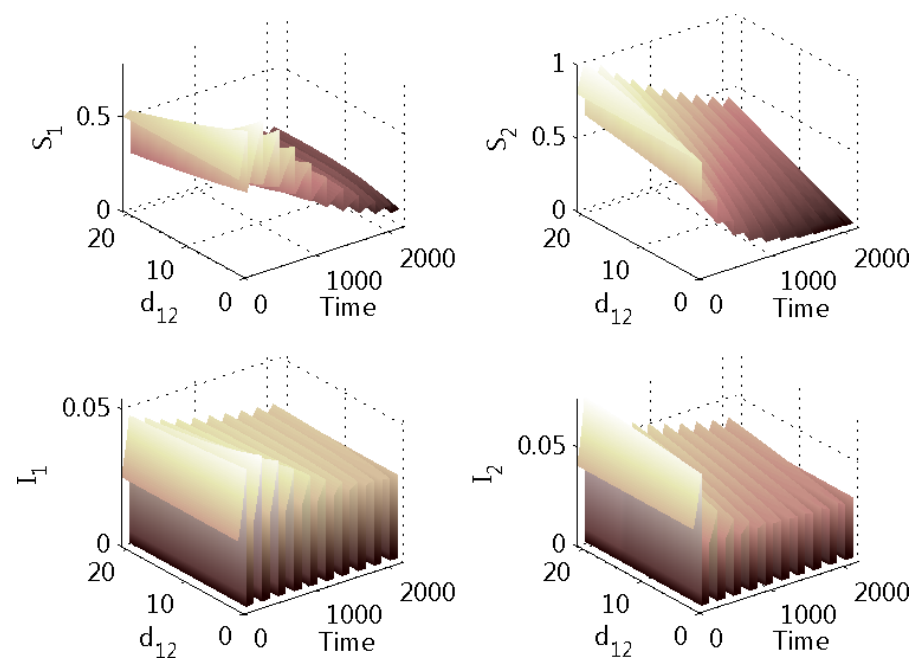

Fig. 3. $g\left(I_{i}\right)=\frac{k I_{i}}{1+\alpha I_{i}}$ and $S_{i} b\left(S_{i}\right)=r_{i} S_{i}\left(1-\frac{S_{i}}{K}\right)^{2}$ for $i=1,2, T=1, l=0.5, d_{21}=0.25$, $D_{12}=0.9, D_{21}=0.8, \delta_{1}=0.25, \delta_{2}=0.2, \delta_{I}=0.9, w_{1}=0.1, w_{2}=0.3, \mu=0.03, r_{1}=0.25$, $r_{2}=0.5, k=2, K=8$. An increase in the dispersal of susceptible pests from a stable patch to an unstable patch has the potential to destabilize the two-patch system.

where one sees that an increase in the dispersal of susceptible pests from the stable patch to the unstable patch has the potential to destabilize $E^{*}$.

In an attempt to understand the nature of the bifurcation of solutions of the single-patch model system (2.2) described by Theorem 2.1, we performed a 
large number of simulations using $g(I)=200 I /(40+I)$ and $b(S)=r-S / 1000$, $T=5, l=0.5, \delta_{1}=0.8 \delta_{I} / 0.99, w=10, \mu=0.03, r=3$, a choice made to have some qualitative similarity to the case of the diamondback moth (high mortality rates for a highly contagious infection, high fecundity for the pest). Note that the period of release of infective pests and of insecticide spraying $(T)$ is kept constant. This reflects the fact that climate or life-cycle rhythms, as well as economic considerations, do not allow for complete freedom in the choice of this period in the field. It would not make sense to spray pesticides every five minutes, for example. The susceptible pest-eradication periodic solution was first allowed to establish itself from initial conditions $S(0)=0$ and $I(0)=\mu$, and then perturbed at $t=400 T$ by adding a fixed number of susceptible pests (setting $S(400 T)=0.0001$ in arbitrary units). The solutions were then followed for another 1000T. Convergence was checked using the highly conservative approach of comparing the values of $S(1400 T)$ and $I(1400 T)$ computed using two different step sizes, differing by a factor of two. If the absolute difference was less than a specified tolerance (0.000001), the simulation was considered to have converged, otherwise the number of steps was increased until reaching a fixed limit. Each colored circle in Fig. 4 is the result of a simulation which passed this convergence check. From these data sets, values at times $n T^{+}$(after addition of infective pests according to $\Delta I(n T)=\mu$ ) were

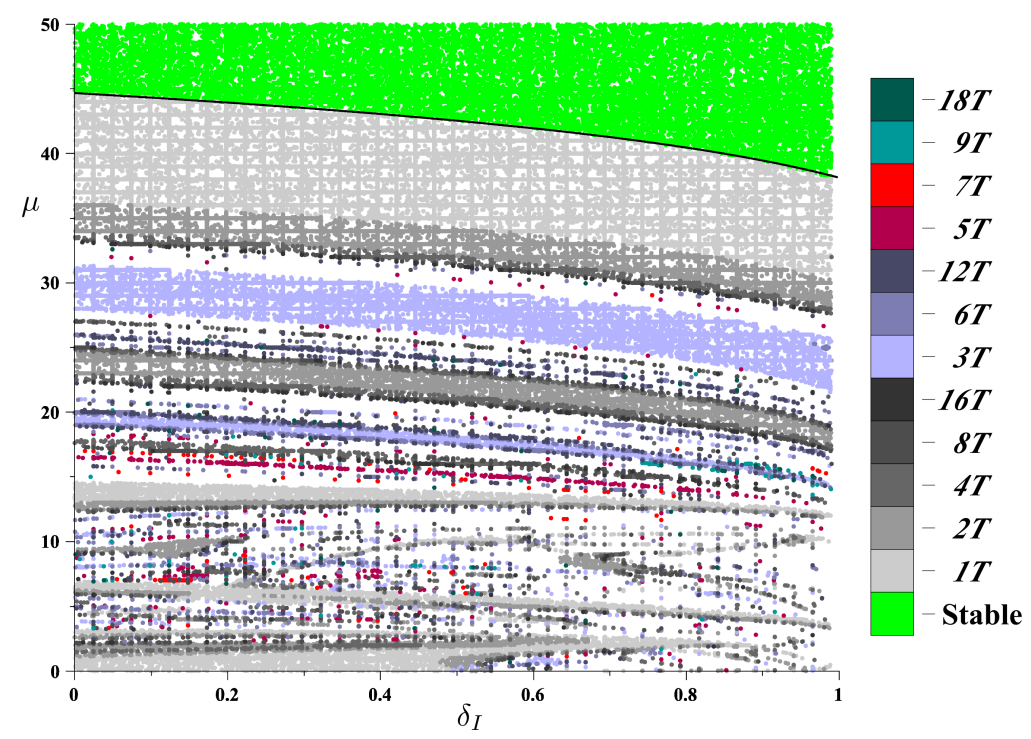

Fig. 4. An illustration of Theorem 2.1 using $g(I)=200 I /(40+I)$ and $b(S)=r-S / 1000, T=5$, $l=0.5, \delta_{1}=0.8 \delta_{I} / 0.99, w=10, \mu=0.03, r=3$. Each point corresponds to one simulation run with initial conditions $S(0)=0$ and $I(0)=\mu$, perturbed at $t=400 T$ by assigning $S(400 T)=$ 0.0001. The green points indicate stability of the susceptible pest-eradication periodic solution. The black curve is the limit case defined by (2.7). The remaining points are coloured according to the periods of the corresponding solutions. White regions indicate failure of convergence of the numerical method, presumably due to the solutions being chaotic. 
recorded. The final 41 such $S I$ pairs (i.e. $S\left(1360 T^{+}\right), S\left(1361 T^{+}\right), \ldots, S\left(1400 T^{+}\right)$ and $\left.I\left(1360 T^{+}\right), I\left(1361 T^{+}\right), \ldots, I\left(1400 T^{+}\right)\right)$were then analysed for periodicity. According to the period detected, a color was chosen for the corresponding colored circle. What one sees is that the susceptible pest-eradication periodic solution is indeed globally asymptotically stable (the green region of Fig. 4) if (2.5) is satisfied, confirming Theorem 2.1 (this is a test of the dedicated numerical software we wrote). Once the limit case (2.7) is passed, we see the predicted bifurcation to a stable positive period solution, confirming Theorem 2.2. What the theorems cannot explain is the remaining structure of the region of instability. Numerically, it seems that period-doubling bifurcations leading to chaos are characteristic of this regime. From a biological point of view, what is interesting to note is that increasing the number of infective pests released $(\mu)$ will, if all other parameters are fixed to the values given above, always lead to control. On the other hand, simply increasing the efficiency of the periodic pesticide spraying (increasing $\delta_{1}$ and $\delta_{I}$, perhaps by simply spraying more pesticide each time) does not typically lead to control.

In a large-scale numerical study, we used the dedicated software written for the bifurcation study above to try to find parameter sets for which the individual patches would be stable $\left(S_{1}\left(700 T^{+}\right)+S_{2}\left(700 T^{+}\right)<0.00003\right)$, but the twopatch system not. One sample parameter set found by this automated search was defined by $\delta_{1}=18 / 25, \delta_{2}=18 / 25, \delta_{I}=891 / 1000, \mu=45.0, r_{1}=2.781782$, $r_{2}=6.245837, d_{12}=0.077159, d_{21}=0.306883, D_{12}=D_{21}=0.1, w_{1}=10.0$, $w_{2}=4.951027, T=5, l=1 / 2, g(I)=I /(30+I / 1000), b_{1}(S)=r_{1}-S / 1000$ and $b_{2}(S)=r_{2}-S / 1000$. Note that this parameter set does not satisfy the relation between death rates and dispersal rates of pests (4.1) we have otherwise enforced for technical reasons. The dispersal rates of susceptibles are asymmetric. Such asymmetry could occur in the wild as the result of prevailing winds or altitude gradients and

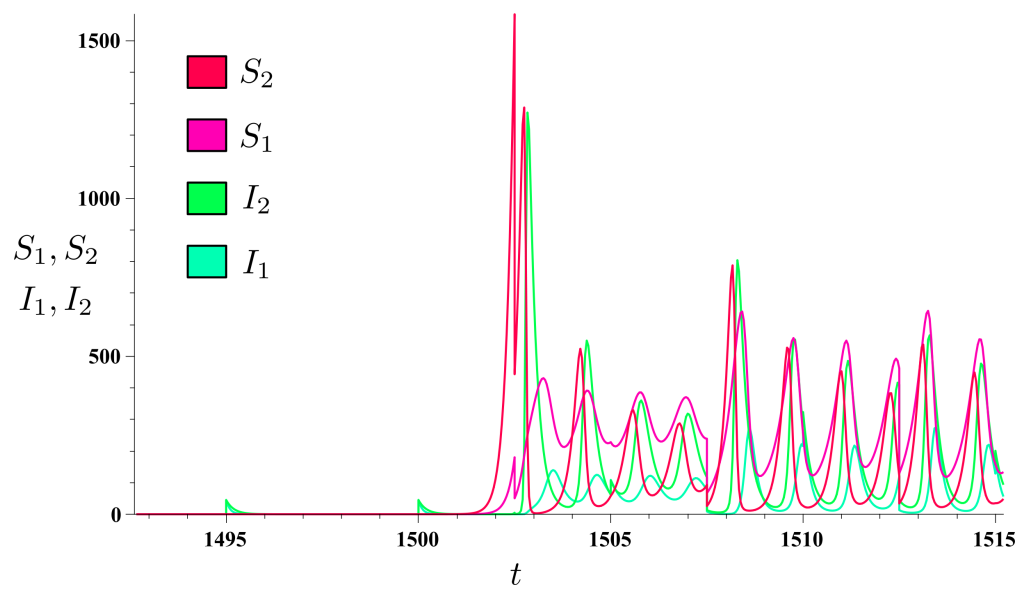

Fig. 5. Instability of the susceptible pest-eradication periodic solution for the two-patch model, in a case in which each patch is stable in isolation. The loss of control is due to dispersion of pests. 
directed water flow, for example. Since the parameter set was found by a numerical simulation performed to hardware precision only (C double precision, corresponding to about 17 significant decimal digits), we also ran high-precision simulations using Maple ${ }^{74}$ computing with 400 decimal digit software floating-point numbers and a seventh-eighth order Runge-Kutta method. These high-precision calculations strongly support the initial calculations, leading us to believe that this parameter set does indeed represent a case in which dispersal between otherwise stable patches can lead to a loss of control for the two-patch system (see Fig. 5). The two-patch solution, once control is lost, appears to be chaotic.

\section{Concluding Remarks}

The focus of this paper is on investigating the behavior of a two-patch integrated pest management model which is subject to a biological control, consisting of the periodic release of infective pests and to a chemical control, consisting of pesticide spraying, which are applied in a periodic fashion, with the same periodicity, but not simultaneously. An abstract, unspecified force of infection, which is possibly nonlinear, is used to describe the spread of the disease which is propagated through the release of infective pests, assuming that both susceptible and infective pests can travel between patches.

Since it is assumed that the infective pests neither damage crops nor reproduce, a measure for the success of the impulsive control strategy is represented by the stability properties of the susceptible pest-eradication periodic solution, which we discussed using Floquet theory for periodic and impulsive systems of ordinary differential equations. In order for our approach to be consistent, a relation between the death rates and the dispersal rates of infective pests has been assumed to hold. Note that, although it has been observed that the case in which infective pests can reproduce with totally successful vertical transmission (i.e., any offspring of an infective pest is an infective pest) can be reformulated to fit our framework, the case of a imperfect vertical transmission (the offspring of infective pests can be both susceptible pests or infective pests) is not treatable using our approach.

The local stability of the susceptible pest-eradication periodic solution is obtained in terms of a condition which is significantly more complicated than its single-patch counterpart in Georgescu and Moroşanu. ${ }^{38}$ This gives formal support to the intuitive notion that the patch structure induces a nontrivial layer of complexity which is worth investigating.

A comparison with the corresponding results in Ref. 39 also reveals the fact that the dispersal of infective pests bears, in some sense, a more prominent role than the dispersal of susceptible pests. Supposing that only the susceptible pests are able to disperse between patches, it has been seen ${ }^{39}$ that the stability or instability of both patches in isolation is transmitted to the system at large regardless of the values of the dispersal rates, in sense that if both patches are stable or unstable in isolation, then the same can be said about the two-patch system. Note that, these findings 
agree with those of Takeuchi, ${ }^{32}$ who observed that, considering a patchy system occupied by a single species, if the species is able to survive at a globally stable equilibrium point when patches are isolated, it will continue to do so at a different equilibrium in the presence of dispersal. However, it has been observed in our setting that the dispersal of infective pests has the potential to destabilize an otherwise stable system, although it cannot do so, given certain technical assumptions, if the death rates of infective pests are the same in each patch. Also, if both patches are unstable in isolation, then the system at large will remain unstable regardless of the values of the dispersal rates of infective pests.

In a system with one stable and one unstable patch, it has been seen numerically that an increase in the dispersal rate of the susceptible pest individuals from the unstable patch to the stable patch has the potential to stabilize the susceptible pest-eradication periodic solution $E^{*}$ and to contribute to the success of the pest control strategy, while an increase in the dispersal rate from the stable patch to the unstable patch has the potential to destabilize $E^{*}$ and to be detrimental to the success of the pest control strategy. Also, in a system with two unstable patches, an increase in the dispersal rate of susceptible pest individuals from one patch to another can have a significant negative impact upon the success of the pest control strategy.

Numerical experiments have revealed complex bifurcation structure in the unstable regime of a single patch, and show that a simple increase in the amount of pesticide applied does not guarantee control of a pest. In a system with two patches, both stable in the absence of dispersion, it has been seen that loss of control in both patches can occur due to dispersal of pests. This was demonstrated in our model both analytically, in the case of equal efficiency of chemical control in both patches, and also in a numerical simulation involving asymmetric dispersion between patches.

\section{Acknowledgments}

The work of P. Georgescu and G. Dimitriu was supported by by CNCSIS UEFISCSU (Romania) project number PN II — IDEI 342/2008, contract no. 447/2009: "Nonlinear evolution equations — theoretical aspects and applications to life and environmental sciences".

\section{References}

1. Alavanja MC, Hoppin JA, Kamel F, Health effects of chronic pesticide exposure: cancer and neurotoxicity, Annu Rev Public Health 25:155-1997, 2004.

2. Kamel F, Hoppin JA, Association of pesticide exposure with neurologic dysfunction and disease, Environ Health Perspect 112:950-958, 2004.

3. Richter ED, Acute human poisonings, Encyclopedia of Pest Management, Pimentel D (ed.), Dekker, New York, pp. 3-6, 2002.

4. Pimentel D, Environmental and economic costs of the application of pesticides primarily in the United States, Environ Dev Sustain 7:229-252, 2005. 
5. Eskenazi B, Chevrier J, Rosas LG, Anderson HA, Bornman MS, Bouwman H, Chen A, Cohn BA, de Jager C, Henshel DS, Leipzig F, Leipzig JS, Lorenz EC, Snedeker SM, Stapleton D, The Pine River statement: human health consequences of DDT use, Environ Health Perspect 117:1359-1367, 2009.

6. Talekar NS, Shelton AM, Biology, Ecology, and management of the diamondback moth, Annu Rev Entomol 38:275-301, 1993.

7. Kogan M, Integrated pest management: historical perspectives and contemporary developments, Annu Rev Entomol 43:243-270, 1998.

8. Smith RF, van den Bosch R, Integrated control, in Pest Control: Biological, Physical and Selected Chemical Methods, Wendell W, Kilglore WW, Doutt RL (eds.), Academic Press, New York, pp. 295-340, 1967.

9. De Maagd RA, Bravo A, Crickmore N, How Bacillus thuringiensis has evolved specific toxins to colonize the insect world, TIG 17:193-199, 2001.

10. Raymond B, Wyres KL, Sheppard SK, Ellis RJ, Bonsall MB, Environmental factors determining the epidemiology and population genetic structure of the Bacillus cereus group in the field, PloS Pathogens 6:e1000905, 2010.

11. DeBach P, Rosen D, Biological Control by Natural Enemies, Cambridge University Press, 1991.

12. Nagai K, Yano E, Predation by Orius sauteri (Poppius) (Heteroptera: Anthocoridae) on Thrips palmi Karny (Thysanoptera: Thripidae). Functional response and selective predation, Appl Entomol Zool 35:565-574, 2000.

13. Port CM, Scopes NEA, Biological control by predatory mites (Phytoseiulus persimilis Athias-Henriot) of red spider mite (Tetranychus urticae Koch) infesting strawberries grown in walk-in plastic tunnels, Plant Pathology 30:95-99, 1981.

14. Grzywacz D, Parnell M, Kibata G, Oduor G, Ogutu W, Miano D, Winstanley D, The development of endemic baculoviruses of Plutella xylostella (diamondback moth, DBM) for control of DBM in East Africa, in The Management of Diamondback Moth and Other Cruciferous Pests, Proceedings of the Fourth International Workshop on Diamondback Moth, Endersby N, Ridland P (eds.), Melbourne University, pp. 179-183, 2001.

15. Brudea V, Modul de acţiune al insecticidelor şi al preparatelor biologice în managementul integrat al dăunătorilor (Romanian), Bucovina Forestieră 1-2:93-100, 2004.

16. Rabb RL, Stinner RE, van den Bosch R, Conservation and augmentation of natural enemies, Theory and Practice of Biological Control, Huffaker CB, Messenger PS (eds.), Academic Press, New York, pp. 233-254, 1976.

17. O'Neil R, Yaninek S, Landis S, Orr D, Biological control and integrated pest management, Integrated Pest Management in the Global Arena, Maredia KM, Dakouo D, Mota-Sanchez D (eds.), Cabi Publishing, Wallingford, pp. 19-30, 2003.

18. Bainov D, Simeonov P, Impulsive Differential Equations: Periodic Solutions and Applications, Longman, John Wiley, New York, NY, 1993.

19. Haddad WM, Chellaboina V, Nersesov SG, Impulsive and Hybrid Dynamical Systems: Stability, Dissipativity and Control, Princeton University Press, Princeton and Oxford, 2006.

20. Yang T, Impulsive Control Theory, Lecture Notes in Control and Information Sciences 272, Springer, Berlin, 2001.

21. Mailleret L, Grognard F, Global stability and optimisation of a general impulsive biological control model, Research Report, INRIA no. 6637, 2008.

22. Zhang H, Chen L, Georgescu P, Impulsive control strategies for pest management, J Biol Syst 15:235-260, 2007.

23. Tang S, Cheke R, State-dependent impulsive models of integrated pest management (IPM) strategies and their dynamic consequences, J Math Biol 50:257-292, 2005. 
24. Tang S, Tang G, Cheke R, Optimum timing for integrated pest management: modelling rates of pesticide application and natural enemy releases, $J$ Theor Biol 264:623-638, 2010.

25. Apreutesei N, Necessary optimality conditions for a Lotka-Volterra three species system, Math Modelling Nat Phenomena 1:123-135, 2006.

26. Apreutesei N, Dimitriu G, Optimal control for Lotka-Volterra systems with a hunter population, Lecture Notes in Computer Science 4818, Springer Verlag, Berlin, 2008, pp. $277-284$.

27. Begon M, Townsend CR, Harper JL, Ecology: From Individuals to Ecosystems, Blackwell Publishing, Oxford, U.K., 2006.

28. Wu J, Modelling dynamics of patchy landscapes: linking metapopulation theory, landscape theory and conservation biology, Wealth, Health and Faith-Sustainability Study in China, Wang R, Zhao J, Ouyang Z, Niu T (eds.), China Environmental Science Press, Beijing, pp. 97-116, 1995.

29. Hauzy C, Hulot FD, Gins A, Loreau M, Intra- and interspecific density-dependent dispersal in an aquatic prey-predator system, J Anim Ecol 76:552-558, 2007.

30. Takeuchi Y, Cui J, Miyazaki R, Saito Y, Permanence of delayed population model with dispersal loss, Math Biosci 201:143-156, 2006.

31. Kuang Y, Takeuchi Y, Predator-prey dynamics in models of prey dispersal in twopatch environments, Math Biosci 120:77-98, 1994.

32. Takeuchi Y, Cooperative system theory and global stability of diffusion models, Acta Appl Math 14:49-57, 1989.

33. Hsieh YH, van den Driessche P, Wang L, Impact of travel between patches for spatial spread of disease, Bull Math Biol 69:1355-1375, 2007.

34. Takeuchi Y, Wang W, Saito Y, Global stability of population models with patch structure, Nonlinear Anal Real World Appl 7:235-247, 2006.

35. Goldwyn E, Hastings A, When can dispersal synchronize populations? Theor Pop Biol 73:395-402, 2008.

36. Briggs C, Hoopes M, Stabilizing effects in spatial parasitoid-host and predator-prey models: a review, Theor Pop Biol 65:299-315, 2004.

37. El Abdllaoui A, Auger P, Kooi B, Bravo de la Parra R, Mchich R, Effects of densitydependent migrations on stability of a two-patch predator-prey model, Math Biosci 210:335-354, 2007.

38. Georgescu P, Moroşanu G, Pest regulation by means of impulsive controls, Appl Math Comput 190:790-803, 2007.

39. Georgescu P, Zhang H, The impulsive control of a two-patch integrated pest management model, Proceedings of 6-th Edition of International Conference on Theory and Applications of Mathematics and Informatics, Breaz D, Breaz N, Wainberg D (eds.), Iaşi, Romania, Acta Univ. Apulensis, Math. Inform. (Special Issue), Aeternitas Publishing House, 2009, pp. 297-320.

40. May RM, Biological populations with nonoverlapping generations: stable points, stable cycles, and chaos, Science 186:645-647, 1974.

41. Pascual M, Diffusion-induced chaos in a spatial predator-prey system, Proc $R$ Soc Lond B 251:1-7, 1993.

42. Koelle K, Vandermeer J, Dispersal-induced desynchronization: from metapopulations to metacommunities, Ecol Lett 8:167-175, 2005.

43. Yang J, Tang S, Effects of population dispersal and impulsive control tactics on pest management, Nonlinear Anal Hybrid Syst 3:487-500, 2009.

44. Liu WM, Hethcote HW, Levin SA, Dynamical behavior of epidemiological models with nonlinear incidence rates, J Math Biol 25:359-380, 1987. 
45. Korobeinikov A, Maini PK, Non-linear incidence and stability of infectious disease models, Math Med Biol 22:113-128, 2005.

46. Capasso V, Serio G, A generalization of Kermack-McKendrick deterministic epidemic model, Math Biosci 42:43-61, 1978.

47. Liu WM, Levin SA, Iwasa Y, Influence of nonlinear incidence rates on the behavior of SIRS epidemiological models, J Math Biol 23:187-204, 1986.

48. Hethcote HW, van den Driessche P, Some epidemiological models with nonlinear incidence, J Math Biol 29:271-287, 1991.

49. Ruan S, Wang W, Dynamical behavior of an epidemic model with a nonlinear incidence rate, J Differential Equations 188:135-163, 2003.

50. Xiao D, Ruan S, Global analysis of an epidemic model with nonmonotone incidence rate, Math Biosci 208:419-429, 2007.

51. Contamine D, Gaumer S, Sigma Rhabdoviruses, Encyclopedia of Virology, 3rd. edn., Volume 4, Mahy BWJ, Van Regenmortel MHV (eds.), Academic Press, Amsterdam, 2008, pp. 576-581.

52. Andreadis TG, Evolutionary strategies and adaptations for survival between mosquito-parasitic microsporidia and their intermediate copepod hosts: a comparative examination of Amblyospora connecticus and Hyalinocysta chapmani (Microsporidia: Amblyosporidae), Folia Parasitologica 52:23-35, 2005.

53. Dodd IB, Shearwin KE, Egan JB, Revisited gene regulation in bacteriophage $\lambda$, Curr Opin Genet Dev 15:145-152, 2005.

54. Chen Y, Evans J, Feldlaufer M, Horizontal and vertical transmission of viruses in the honey bee, Apis mellifera, J Invertebr Pathol 92:152-159, 2006.

55. Bouchon D, Rigaud T, Juchault P, Evidence for widespread Wolbachia infection in isopod crustaceans: molecular identification and host feminization, Proc Biol Sci 265:1081-1090, 1998.

56. Cory JS, Myers JH, The ecology and evolution of insect baculoviruses, Annu Rev Ecol Evol Systemat 34:239-272, 2003.

57. Nordin GL, Brown GC, Jackson DM, Vertical transmission of two baculoviruses infectious to the tobacco budworm, Heliothis virescens (F.) (Lepidoptera: Noctuidae) using an autodissemination technique, $J$ Kansas Entomol Soc 63:393-398, 1990.

58. Stewart AD, Logsdon JM, Kelley SE, An empirical study of the evolution of virulence under both horizontal and vertical transmission, Evolution 59:730-739, 2005.

59. Lambrechts L, Scott TW, Mode of transmission and the evolution of arbovirus virulence in mosquito vectors, Proc R Soc B 276:1369-1378, 2009.

60. Dunn AM, Smith JE, Microsporidian life cycles and diversity: the relationship between virulence and transmission, Microb Infect 3:381-388, 2001.

61. Talekar NS, Biological control of diamondback moth in Taiwan - a review, Plant Prot Bull 38:167-189, 1996.

62. Rondon SI, The Potato Tuberworm: a literature review of its biology, ecology, and control, Am J Pot Res 87:149-166, 2010.

63. Hilton S, Baculoviruses: molecular biology of ganuloviruses, Encyclopedia of Virology, 3rd. edn., Volume 1, Mahy BWJ, Van Regenmortel MHV (eds.), Academic Press, Amsterdam, 2008, pp. 211-219.

64. Dezianian A, Sajap AS, Lau WH, Omar D, Kadir HA, Mohamed R, Yusoh MRM, Morphological characteristics of P. xylostella granulovirus and effects on its larval host diamondback moth Plutella xylostella L. (Lepidoptera, Plutellidae), Am J Agric Bio Sci 5:43-49, 2010. 
65. Arthurs SP, Lacey LA, De La Rosa F, Evaluation of a Granulovirus (PoGV) and Bacillus thuringiensis subsp. kurstaki for control of the potato tuberworm (Lepidoptera: Gelechiidae) in stored tubers, J Econ Entomol 101:1540-1546, 2008.

66. Volkman LE, Baculoviruses: Pathogenesis, in Encyclopedia of Virology, 3rd. edn., Volume 1, Mahy BWJ, Van Regenmortel MHV (eds.), Academic Press, Amsterdam, 2008, pp. 265-272.

67. Nakai M, Goto C, Shiotsuki T, Kunimi Y, Granulovirus prevents pupation and retards development of Adoxophyes honmai larvae, Physiol Entomol 27:157-164, 2002.

68. Hoy MA, Myths, models and mitigation of resistance to pesticides, Phil Trans $R$ Soc Lond B 353:1787-1795, 1998.

69. Lee Y, Fuxa JR, Transport of wild-type and recombinant nucleopolyhedroviruses by scavenging and predatory arthropods, Microb Ecol 39:301-313, 2000.

70. Georgescu P, Zhang H, Chen L, Bifurcation of nontrivial periodic solutions for an impulsively controlled pest management model, Appl Math Comput 202:675-687, 2008.

71. Bacaër N, Ouifki R, Growth rate and basic reproduction number for population models with a simple periodic factor, Math Biosci 210: 647-658, 2007.

72. Bacaër N, Periodic matrix population models: growth rate, basic reproduction number and entropy, Bull Math Biol 71: 1781-1792, 2009.

73. Wang W, Zhao XQ, Threshold dynamics for compartmental epidemic models in periodic environments, J Dyn Diff Eq 20:699-717, 2008.

74. Monagan MB, Geddes KO, Heal KM, Labahn G, Vorkoetter SM, McCarron J, DeMarco P, Maple 10 Programming Guide, Maplesoft, Waterloo ON, Canada, 2005.

\section{Appendix: The Exponential Representation Formula}

To write the fundamental matrix $\Phi(t)$ of (2.1) as a matrix exponential, we first state several considerations regarding the exponential representation formula for the solutions of a $n$-dimensional time-dependent homogeneous linear differential system.

Let us consider the differential system with continuous coefficients

$$
x^{\prime}(t)=B(t) x(t), \quad t \geq 0 .
$$

The fundamental matrix of (A.1) which satisfies $\Phi(0)=I_{n}$ can be expressed as a Peano-Baker series of the form,

$$
\begin{aligned}
\Phi(t)= & I_{n}+\int_{0}^{t} B\left(s_{1}\right) d s_{1}+\int_{0}^{t} B\left(s_{1}\right) \int_{0}^{s_{1}} B\left(s_{2}\right) d s_{2} d s_{1} \\
& +\int_{0}^{t} B\left(s_{1}\right) \int_{0}^{s_{1}} B\left(s_{2}\right) \int_{0}^{s_{2}} B\left(s_{3}\right) d s_{3} d s_{2} d s_{1}+\cdots
\end{aligned}
$$

To compute a monodromy matrix, a closed form representation of $\Phi$ would be significantly more useful. If $B$ commutes with its integral, i.e.,

$$
B(t)\left(\int_{0}^{t} B(s) d s\right)=\left(\int_{0}^{t} B(s) d s\right) B(t) \quad \text { for } t \geq 0,
$$


then the fundamental matrix $\Phi$ can be expressed as the exponential of a timedependent matrix, in the form,

$$
\Phi(t)=\exp \left(\int_{0}^{t} B(s) d s\right),
$$

where, given $N \in M_{n}(\mathbb{R})$, $\exp (N)$ is defined as,

$$
\exp (N)=\sum_{k=0}^{\infty} \frac{1}{k !} N^{k}
$$

Note that, in general, the fundamental matrix $\Phi$ given by (A.2) may be different from $\exp \left(\int_{0}^{t} B(s) d s\right)$ if $B$ does not satisfy (A.3). Also, it is easy to see that (A.3) holds if $B(t)$ commutes with $B(s)$ for all $t, s \geq 0$, i.e.,

$$
B(t) B(s)=B(s) B(t) \text { for } t, s \geq 0 .
$$

In this regard, note that the applicability of the results in Yang and Tang ${ }^{43}$ should be somewhat restricted, as no commutation condition is verified therein.

\section{Appendix B: The Proof of Lemma 4.1}

Proof. Let $C=\left(\begin{array}{cc}-\left(w_{1}+D_{12}\right) & D_{21} \\ D_{12} & -\left(w_{2}+D_{21}\right)\end{array}\right)$ and let us also denote by $\left(I_{1}^{*}, I_{2}^{*}\right)^{t}$ the desired periodic solution of (4.2). Obviously, one should have

$$
\left(\begin{array}{l}
I_{1}^{*}(t) \\
I_{2}^{*}(t)
\end{array}\right)= \begin{cases}\exp (t C)\left(\begin{array}{c}
I_{1}^{*}(0+) \\
I_{2}^{*}(0+)
\end{array}\right), & t \in(0, l T] \\
\left(1-\delta_{I}\right) \exp (t C)\left(\begin{array}{l}
I_{1}^{*}(0+) \\
I_{2}^{*}(0+)
\end{array}\right), & t \in(l T, T] .\end{cases}
$$

By the $T$-periodicity requirement, it is seen that the initial data should satisfy

$$
\left(1-\delta_{I}\right) e^{T C}\left(\begin{array}{c}
I_{1}^{*}(0+) \\
I_{2}^{*}(0+)
\end{array}\right)+\left(\begin{array}{c}
\mu \\
\mu
\end{array}\right)=\left(\begin{array}{c}
I_{1}^{*}(0+) \\
I_{2}^{*}(0+)
\end{array}\right) .
$$

Let

$$
\lambda_{1,2}=\frac{-\left[\left(w_{1}+D_{12}\right)+\left(w_{2}+D_{21}\right)\right] \pm \sqrt{\left[\left(w_{1}+D_{12}\right)-\left(w_{2}+D_{21}\right)\right]^{2}+4 D_{12} D_{21}}}{2}
$$

be the (real) eigenvalues of $C$. Since

$$
\left\{\begin{array}{l}
\lambda_{1}+\lambda_{2}=-\left(w_{1}+D_{12}\right)-\left(w_{2}+D_{21}\right)<0 \\
\lambda_{1} \cdot \lambda_{2}=\left(w_{1}+D_{12}\right)\left(w_{2}+D_{21}\right)-D_{12} D_{21}>0
\end{array}\right.
$$

it follows that $\lambda_{1}, \lambda_{2}<0$. Consequently, the eigenvalues of $\exp (T C)$ are $e^{\lambda_{1} T}$, $e^{\lambda_{2} T} \in(0,1)$, which implies that $I_{2}-\left(1-\delta_{I}\right) \exp (T C)$ is invertible, and

$$
\left(\begin{array}{c}
I_{1}^{*}(0+) \\
I_{2}^{*}(0+)
\end{array}\right)=\left(I_{2}-\left(1-\delta_{I}\right) \exp (T C)\right)^{-1}\left(\begin{array}{c}
\mu \\
\mu
\end{array}\right) .
$$


Now, it is seen that $I_{1}^{*}-I_{2}^{*}$ verifies

$$
\left\{\begin{aligned}
\left(I_{1}^{*}-I_{2}^{*}\right)^{\prime}(t) & =-\left(w_{1}+2 D_{12}\right)\left(I_{1}^{*}-I_{2}^{*}\right)(t), & & t \neq(n+l-1) T, \quad t \neq n T ; \\
\Delta\left(I_{1}^{*}-I_{2}^{*}\right)(t) & =-\delta_{I}\left(I_{1}^{*}-I_{2}^{*}\right)(t), & & t=(n+l-1) T ; \\
\Delta\left(I_{1}^{*}-I_{2}^{*}\right)(t) & =0, & & t=n T,
\end{aligned}\right.
$$

which implies that $\lim _{t \rightarrow \infty}\left(I_{1}^{*}(t)-I_{2}^{*}(t)\right)=0$ and, since $I_{1}^{*}, I_{2}^{*}$ are $T$-periodic, $I_{1}^{*} \equiv$ $I_{2}^{*} \equiv I^{*}$. Now, $I^{*}$ verifies

$$
\left\{\begin{aligned}
I^{*} & =-\frac{w_{1}+w_{2}}{2} I^{*}, & & t \neq(n+l-1) T, \quad t \neq n T \\
\Delta I^{*} & =-\delta_{I} I^{*}, & & t=(n+l-1) T ; \\
\Delta I^{*} & =\mu, & t & =n T
\end{aligned}\right.
$$

and the periodicity condition (B.2) translates as,

$$
\left(1-\delta_{I}\right) e^{-\frac{w_{1}+w_{2}}{2} T} I^{*}(0+)+\mu=I^{*}(0+),
$$

which implies that

$$
I^{*}(0+)=\frac{\mu}{1-\left(1-\delta_{I}\right) e^{-\frac{w_{1}+w_{2}}{2} T}} .
$$

Consequently, $I^{*}$ is given by,

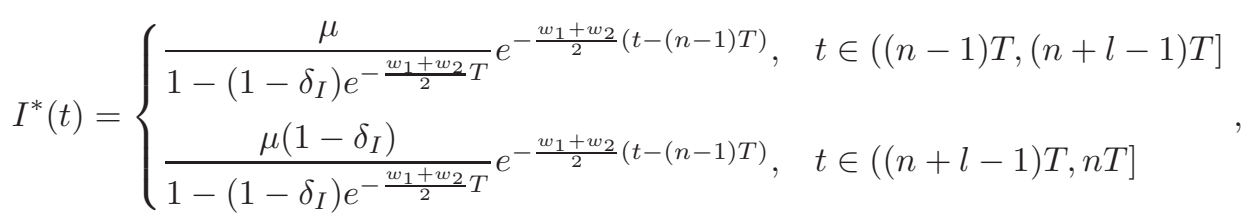

and the susceptible pest-eradication periodic solution $E^{*}$ of $(2.1)$ is given by,

$$
E^{*}=\left(0,0, I^{*}, I^{*}\right)
$$

Now let $\left(I_{1}, I_{2}\right)^{t}$ be a solution of (4.2). As $\left(I_{1}-I^{*}, I_{2}-I^{*}\right)^{t}$ satisfies the system

$$
\begin{cases}\left(\begin{array}{c}
I_{1}-I^{*} \\
I_{2}-I^{*}
\end{array}\right)^{\prime}(t)=C\left(\begin{array}{c}
I_{1}-I^{*} \\
I_{2}-I^{*}
\end{array}\right)(t), & t \neq(n+l-1) T, \quad t \neq n T ; \\
\Delta\left(\begin{array}{c}
I_{1}-I^{*} \\
I_{2}-I^{*}
\end{array}\right)(t)=\left(1-\delta_{I}\right)\left(\begin{array}{c}
I_{1}-I^{*} \\
I_{2}-I^{*}
\end{array}\right)(t), & t=(n+l-1) T ; \\
\Delta\left(\begin{array}{c}
I_{1}-I^{*} \\
I_{2}-I^{*}
\end{array}\right)(t)=0, & t=n T\end{cases}
$$


it is seen that,

$$
\left(\begin{array}{l}
I_{1}-I^{*} \\
I_{2}-I^{*}
\end{array}\right)(t)= \begin{cases}\left(1-\delta_{I}\right)^{n-1} \exp (t C)\left(\begin{array}{c}
I_{1}^{0}-I^{*}(0+) \\
I_{2}^{0}-I^{*}(0+)
\end{array}\right), & t \in((n-1) T,(n+l-1) T] \\
\left(1-\delta_{I}\right)^{n} \exp (t C)\left(\begin{array}{c}
I_{1}^{0}-I^{*}(0+) \\
I_{2}^{0}-I^{*}(0+)
\end{array}\right), & t \in((n+l-1) T, n T] .\end{cases}
$$

Since the eigenvalues of $\exp (T C)$ are $e^{\lambda_{1} T}, e^{\lambda_{2} T} \in(0,1)$ and $\exp ((t-(n-1) T) C)$ is bounded for $t \in((n-1) T, n T]$, it is seen that,

$$
\lim _{n \rightarrow \infty}\left\|\left(1-\delta_{I}\right)^{n-1} \exp ((n-1) T C)\right\|=0
$$

for any matrix norm $\|\cdot\|$, from which the conclusion follows.

\section{Appendix C: The Proof of the Commutation Condition}

It is seen that

$$
\begin{aligned}
& A(t)\left(\int_{0}^{t} A(s) d s\right)=\left(\begin{array}{ll}
a_{1}(t) \int_{0}^{t} a_{1}(s) d s+d_{12} d_{21} t & d_{21}\left(t a_{1}(t)+\int_{0}^{t} a_{2}(s) d s\right) \\
d_{12}\left(t a_{2}(t)+\int_{0}^{t} a_{1}(s) d s\right) & a_{2}(t) \int_{0}^{t} a_{2}(s) d s+d_{12} d_{21} t
\end{array}\right), \\
& \left(\int_{0}^{t} A(s) d s\right) A(t)=\left(\begin{array}{ll}
a_{1}(t) \int_{0}^{t} a_{1}(s) d s+d_{12} d_{21} t & d_{12}\left(t a_{2}(t)+\int_{0}^{t} a_{1}(s) d s\right) \\
d_{21}\left(t a_{1}(t)+\int_{0}^{t} a_{2}(s) d s\right) & a_{2}(t) \int_{0}^{t} a_{2}(s) d s+d_{12} d_{21} t
\end{array}\right)
\end{aligned}
$$

with

$$
\begin{aligned}
& a_{1}(t)=\left(r_{1}-d_{12}\right)-g\left(I^{*}(t)\right) \\
& a_{2}(t)=\left(r_{2}-d_{21}\right)-g\left(I^{*}(t)\right) .
\end{aligned}
$$

Also,

$$
\begin{aligned}
t a_{2}(t)+\int_{0}^{t} a_{1}(s) d s & =t\left(r_{1}-d_{12}+r_{2}-d_{21}\right)-t g\left(I^{*}(t)\right)-\int_{0}^{t} g\left(I^{*}(s)\right) d s \\
& =t a_{1}(t)+\int_{0}^{t} a_{2}(s) d s
\end{aligned}
$$

from which we deduce that

$$
A(t)\left(\int_{0}^{t} A(s) d s\right)=\left(\int_{0}^{t} A(s) d s\right) A(t), \quad \forall t \geq 0 .
$$

This commutation condition represents the motivation for using the same nonlinear force of infection $g$ and the same proportional loss of infective pests $\delta_{I}$ in each patch and also for using condition (4.1), meant to ensure that the limiting susceptible 
pest-eradication periodic solution has identical components in each patch. In this regard, if different nonlinear forces of infection $g_{1}$ and $g_{2}$ were used in each patch and the susceptible pest-eradication periodic solution had distinct components $I_{1}^{*}$ and $I_{2}^{*}$ for each patch, then the desired equality

$$
t\left(g_{1}\left(I_{1}^{*}(t)\right)-g_{2}\left(I_{2}^{*}(t)\right)\right)=\int_{0}^{t}\left(g_{1}\left(I_{1}^{*}(s)\right)-g_{2}\left(I_{2}^{*}(s)\right)\right) d s \quad \forall t \geq 0
$$

would not necessarily hold, as this would imply that $t \mapsto g_{1}\left(I_{1}^{*}(t)\right)-g_{2}\left(I_{2}^{*}(t)\right)$ is a constant function. Now, for $g_{1}=g_{2}=g$ and $I_{1}^{*}=I_{2}^{*}=I^{*}$, it is seen from (4.2) that $I^{*}$ satisfies

$$
\left\{\begin{array}{l}
\left(I^{*}\right)^{\prime}(t)=\left(-w_{1}+D_{21}-D_{12}\right) I^{*}(t) \quad t \neq(n+l-1) T, t \neq n T \\
\left(I^{*}\right)^{\prime}(t)=\left(-w_{2}+D_{12}-D_{21}\right) I^{*}(t)
\end{array}\right.
$$

from which we deduce that $-w_{1}+D_{21}-D_{12}=-w_{2}+D_{12}-D_{21}$, i.e. (4.1) holds. 